\title{
The Galactic Center Molecular Cloud Survey
}

\section{A steep linewidth-size relation and suppression of star formation}

\author{
Jens Kauffmann ${ }^{1}$, Thushara Pillai ${ }^{1}$, Qizhou Zhang ${ }^{2}$, Karl M. Menten ${ }^{1}$, Paul F. Goldsmith ${ }^{3}$, \\ Xing $\mathrm{Lu}^{2,4}$, and Andrés E. Guzmán ${ }^{5}$
}

\author{
1 Max-Planck-Institut für Radioastronomie, Auf dem Hügel 69, 53121 Bonn, Germany \\ e-mail: jens. kauffmann@gmail.com \\ 2 Harvard-Smithsonian Center for Astrophysics, 60 Garden Street, Cambridge, MA 02138, USA \\ 3 Jet Propulsion Laboratory, California Institute of Technology, 4800 Oak Grove Drive, Pasadena, CA 91109, USA \\ 4 School of Astronomy and Space Science, Nanjing University, 22 Hankou Road, 210093 Nanjing, PR China \\ 5 Departamento de Astronomía, Universidad de Chile, Camino el Observatorio 1515, Las Condes, 1058 Santiago, Chile
}

Received 7 January 2016 / Accepted 27 August 2016

\begin{abstract}
The central molecular zone (CMZ; inner $\sim 200 \mathrm{pc}$ ) of the Milky Way is a star formation (SF) environment with very extreme physical properties. Exploration of SF in this region is important because (i) this region allows us to test models of star formation under exceptional conditions; and (ii) the CMZ clouds might be suitable to serve as templates to understand the physics of starburst galaxies in the nearby and the distant universe. For this reason we launched the Galactic Center Molecular Cloud Survey (GCMS), the first systematic study that resolves all major CMZ clouds at interferometer angular resolution (i.e., a few arc seconds). Here we present initial results based on observations with the Submillimeter Array (SMA) and the Atacama Pathfinder Experiment (APEX). Our study is complemented by dust emission data from the Herschel Space Telescope and a comprehensive literature survey of CMZ star formation activity. Our research reveals (i) an unusually steep linewidth-size relation, $\sigma(v) \propto r_{\text {eff }}^{0.66 \pm 0.18}$, down to velocity dispersions $\sim 0.6 \mathrm{~km} \mathrm{~s}^{-1}$ at $0.1 \mathrm{pc}$ scale. This scaling law potentially results from the decay of gas motions to transonic velocities in strong shocks. The data also show that, relative to dense gas in the solar neighborhood, (ii) star formation is suppressed by factors $\gtrsim 10$ in individual CMZ clouds. This observation encourages exploration of processes that can suppress SF inside dense clouds for a significant period of time.
\end{abstract}

Key words. ISM: clouds - methods: data analysis - stars: formation

\section{Introduction}

The central molecular zone (CMZ), the inner $\sim 200 \mathrm{pc}$ of our Galaxy, is a star-forming environment with extreme physical properties. About $3-10 \%$ of the total molecular gas and star formation (SF) of the Milky Way ${ }^{1}$ reside at $|\ell| \leq 3^{\circ}$ (i.e., within galactocentric radii $\leq 430 \mathrm{pc}$ ). CMZ molecular clouds have unusually high mean $\mathrm{H}_{2}$ densities $\sim 10^{4} \mathrm{~cm}^{-3}$ and column densities $\sim 10^{23} \mathrm{~cm}^{-2}$ (e.g., Lis \& Carlstrom 1994), and they are subject to an average gas pressure from hot gas of order $10^{6}$ to $7 \mathrm{~K} \mathrm{~cm}^{-3}$ (Yamauchi et al. 1990; Spergel \& Blitz 1992; Muno et al. 2004). The gas is pervaded by a strong magnetic field of a few $10^{3} \mu \mathrm{G}$ (Yusef-Zadeh et al. 1984; Uchida et al. 1985; Chuss et al. 2003; Novak et al. 2003) that also penetrates the CMZ clouds (Pillai et al. 2015). Unusually wide lines $\gtrsim 10 \mathrm{~km} \mathrm{~s}^{-1}$ on spatial scales $\gtrsim 1$ pc (e.g., Güsten et al. 1981, strong and widespread $\mathrm{SiO}$ emission tracing shocks

\footnotetext{
1 Observations of line and dust emission indicate that the $|\ell| \leq 3^{\circ}$ region contains of order $(3$ to 8$) \times 10^{7} M_{\odot}$ of molecular gas (Dahmen et al. 1998; Tsuboi et al. 1999; Longmore et al. 2013a). It forms stars at a rate of order $0.1 M_{\odot} \mathrm{yr}^{-1}$ (Yusef-Zadeh et al. 2009; Immer et al. 2012b; Longmore et al. 2013a; Koepferl et al. 2015). The Milky Way contains $(1.0 \pm 0.3) \times 10^{9} M_{\odot}$ of molecular gas (Heyer \& Dame 2015), while the star-formation rate is 1 to $4 M_{\odot} \mathrm{yr}^{-1}$ (Diehl et al. 2006; Misiriotis et al. 2006; Lee et al. 2012).
}

(Martín-Pintado et al. 1997; Hüttemeister et al. 1998; Riquelme et al. 2010b), prevalence of molecules likely ejected from grain surfaces via shocks (Requena-Torres et al. 2006, 2008), and collisionally-excited methanol masers (Mills et al. 2015; also see Menten et al. 2009, though) suggest that much of the gas in the CMZ is subject to violent gas motions, such as cloud-cloud collisions at high velocities. Gas temperatures are typically in the range 50 to $100 \mathrm{~K}$ (Güsten et al. 1981; Hüttemeister et al. 1993; Ao et al. 2013; Mills \& Morris 2013; Ott et al. 2014; Ginsburg et al. 2016; also see Riquelme et al. 2010a, 2012). In stark contrast to spiral-arm clouds, and despite relatively high gas densities, the high gas temperature in the CMZ is mysteriously decoupled from the much lower temperature of dust grains, for which values $\sim 20 \mathrm{~K}$ are obtained (e.g., Güsten et al. 1981; Molinari et al. 2011; Longmore et al. 2012; see Clark et al. 2013, for some modeling work). The $\mathrm{CMZ}$ is populated by numerous exotic objects, such as masers that are possibly enabled by the extreme physical conditions of the environment (e.g., Ginsburg et al. 2015). The clouds reside at a distance of $8.34 \pm 0.16 \mathrm{kpc}$ (Reid et al. 2014; this value is adopted throughout this study), and they appear to lie on a well-organized common orbit (e.g., Molinari et al. 2011 and Kruijssen et al. 2015) along which clouds might also systematically evolve (Longmore et al. 2013b). 
The study of CMZ molecular clouds is critical for two reasons. First, the CMZ allows to explore an extreme point in the star formation parameter space: valid models of SF physics must describe star formation in the CMZ environment as well as under the conditions prevailing in clouds closer to sun. Second, the $\mathrm{CMZ}$ might serve as a template for unresolved processes that are active in nearby and more distant starburst galaxies.

For this reason we launched the Galactic Center Molecular Cloud Survey (GCMS), the first systematic study resolving all major $\mathrm{CMZ}$ molecular clouds at interferometer angular resolution. This work is inspired by first interferometric investigations of the $\mathrm{CMZ}$ cloud G0.253+0.016 ${ }^{2}$ (Güsten et al. 1981; Lis et al. 1994, 2001; Lis \& Menten 1998; a.k.a. the "Brick": Longmore et al. 2012) with the Submillimeter Array (SMA; Kauffmann et al. 2013b and Johnston et al. 2014) and ALMA (Rathborne et al. 2014b, 2015). Section 2 introduces the GCMS. Some of the observations were already explored by Kauffmann et al. (2013b), Kendrew et al. (2013), and Lu et al. (2015). Here we present a first study that characterizes all targets in a homogeneous way and permits to draw general conclusions about the structure of CMZ molecular clouds.

Our study employs observations of dust and the $\mathrm{N}_{2} \mathrm{H}^{+}(3-2)$ transition. We have made this choice because these probes are believed to be faithful tracers of the densest gas in molecular clouds (Sect. 2.2 summarizes properties of $\mathrm{N}_{2} \mathrm{H}^{+}$).

In this paper we focus on two particular topics. First, we conduct a systematic exploration of the kinematics of CMZ molecular clouds. In Kauffmann et al. (2013b) we demonstrated for the first time that line widths $\lesssim 1 \mathrm{~km} \mathrm{~s}^{-1}$ prevail in some CMZ cloud fragments on spatial scales $\lesssim 0.1 \mathrm{pc}$. This is remarkable given the much larger aforementioned line widths $\gtrsim 10 \mathrm{~km} \mathrm{~s}^{-1}$ on spatial scales $\gtrsim 1$ pc. In essence this exploration of cloud kinematics over a range of spatial scales constrains the linewidth-size relation (e.g., Larson 1981). While this concept has been explored extensively for entire regular Milky Way clouds (starting with, e.g., Sanders et al. 1985 and Solomon et al. 1987), relatively little work has been done on CMZ clouds (e.g., Oka et al. 1998, Miyazaki \& Tsuboi 2000, and Shetty et al. 2012). These papers find that the velocity dispersion scales with spatial scale as $\sigma(v) \propto \ell^{h_{\sigma(v)}}$, where the linewidth-size slope, $h_{\sigma(v)}$, lies in the range 0.4 to 0.8 , depending on the author, method, and emission line studied. The studies agree, though, that $h_{\sigma(v)}$ appears to be the same inside and outside the CMZ. Here we come to a different conclusion because of the small line widths now revealed by the interferometer data on spatial scales $\lesssim 0.1 \mathrm{pc}$.

Second, we explore the star-formation rates of individual molecular clouds in the CMZ. One of the surprising trends in the CMZ is that, despite the high average gas densities, the starformation rate per unit dense gas is suppressed when compared to the solar neighborhood. For example, Güsten \& Downes (1983) and Caswell et al. (1983) found early on that very few $\mathrm{H}_{2} \mathrm{O}$ masers reside in the $\mathrm{CMZ}$, relative to the high mass of dense gas. Caswell (1996) later showed the same for class II methanol masers that uniquely trace high-mass star formation. Taylor et al. (1993) concluded that, based on the mass and density of molecular material, the $\mathrm{CMZ}$ should contain about an order of magnitude more $\mathrm{H}_{2} \mathrm{O}$ masers than observed. Observations of the dense gas with increased angular resolution at wavelengths $\lambda \lesssim 1 \mathrm{~mm}$ eventually revealed individual clouds with little star formation (Lis et al. 1994, 2001; Lis \& Menten 1998). The most recent

\footnotetext{
2 Observations of G0.253+0.016 with the Midcourse Space Experiment (MSX) inspired Egan et al. (1998) and Carey et al. (1998) to coin the now-popular term of the Infrared Dark Cloud (IRDC).
}

studies established that SF relations that apply in the solar neighborhood (Heiderman et al. 2010; Lada et al. 2010; Evans et al. 2014) overpredict CMZ star formation by an order of magnitude, both on the scale of the entire CMZ (Longmore et al. 2013a) and on that of individual clouds (Kauffmann et al. 2013b). Here we expand this research by obtaining individual star-formation rates for all major CMZ molecular clouds. One interesting hypothesis is that $\mathrm{CMZ}$ star formation in the dense gas is suppressed because all CMZ molecular clouds are young and are in a state that precedes efficient star formation. Our new data on individual clouds allow to test and reject this scenario.

A number of aspects can be explored with our data and the GCMS results will be published in a series of papers. We therefore also include a broad description of the data set in this initial paper. For example, a companion study published simultaneously with the current work (Kauffmann et al. 2017, hereafter Paper II) explores the density structure of CMZ clouds and explores its relationship with the observed star formation activity.

The current paper is organized as follows. Section 2 describes the sample, the observations, and the data reduction. In Sect. 3 we characterize and analyze the kinematic structure of CMZ molecular clouds. The star formation characteristics of the clouds are then discussed in Sect. 4. General conclusions on the structure of CMZ molecular clouds are drawn in Sect. 5. A summary of the study is provided in Sect. 6. The work in the paper is supported by appendices on imaging of interferometer data (Appendix A), observations of CMZ star formation (Appendix B), and methods to determine the $\mathrm{CMZ}$ star-formation rate (Appendix $\mathrm{C}$ ).

\section{Sample, observations, and data reduction}

\subsection{Sample selection}

Previous research into G0.253+0.016 demonstrates that investigating massive and dense CMZ molecular clouds informs both general studies of star formation and research into the processes acting in the CMZ. The GCMS is therefore designed to provide a general overview of the physical conditions in dense and massive CMZ molecular clouds resembling G0.253+0.016. Figure 1 and Table 1 give an overview of the regions studied here, including some ancillary clouds which we characterize using archival data. The masses and radii listed in Table 1 are derived in Sect. 2.4 using Herschel data. We select target regions on the basis of SCUBA $850 \mu \mathrm{m}$ dust emission maps (Pierce-Price et al. 2000) collected from the SCUBA Legacy Catalogue archive ${ }^{3}$ (Di Francesco et al. 2008; the beam size is $19^{\prime \prime}$ at $850 \mu \mathrm{m}$ wavelength). Manual experimentation reveals that the SCUBA $850 \mu \mathrm{m}$ contour at $5 \mathrm{Jy}$ beam $^{-1}$ traces the outline of G0.253+0.016 very well. Further targets are therefore selected by searching the maps for clouds exceeding the $5 \mathrm{Jy} \mathrm{beam}^{-1}$ intensity threshold. Assuming emission from dust at a temperature of $20 \mathrm{~K}$ and opacities following Ossenkopf \& Henning (1994), this contour corresponds to an $\mathrm{H}_{2}$ column density $\sim 2 \times 10^{23} \mathrm{~cm}^{-2}$ (Kauffmann et al. 2008).

We limit our study to the region between Sgr C and Sgr B2. Target regions are further required to be large enough to sample a significant mass reservoir. This selection is done manually by visual inspection of the target sizes. The least massive region in our sample contains a mass of $2.5 \times 10^{4} M_{\odot}$. Our survey is effectively complete to clouds exceeding this mass. We further exclude regions too large to be mapped in reasonable time. Only

\footnotetext{
http://www2 . cadc-ccda.hia-iha.nrc-cnrc.gc.ca/ community/scubalegacy/
} 
J. Kauffmann et al.: The Galactic Center Molecular Cloud Survey. I.

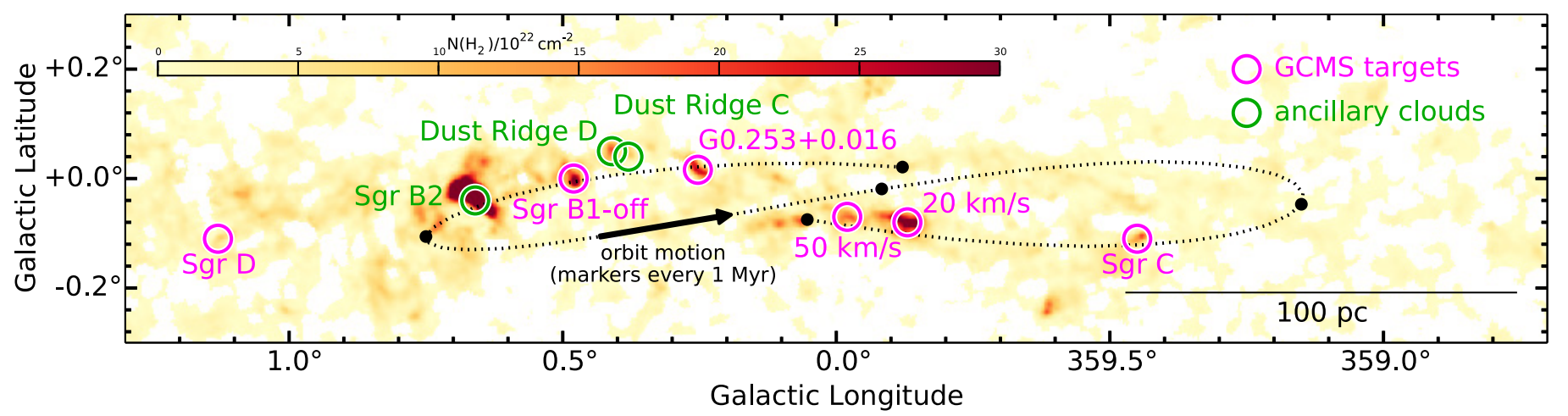

Fig. 1. Overview of the inner CMZ. Clouds targeted by GCMS interferometer observations presented here are highlighted in magenta. Other ancillary clouds discussed in this paper are shown in green. A dotted line shows part of the orbit for CMZ clouds proposed by Kruijssen et al. (2015). The background image presents a column density map derived from Herschel dust emission data, as described in this paper.

Table 1. Basic information on the clouds discussed in this paper.

\begin{tabular}{|c|c|c|c|c|c|}
\hline Target & Location $(\ell, b)$ & $\begin{array}{l}\text { Radius } \\
\text { pc }\end{array}$ & $\begin{array}{l}\text { Mass } \\
10^{4} M_{\odot}\end{array}$ & $\begin{array}{l}\text { Peak mass per beam } \\
10^{3} M_{\odot}\end{array}$ & $\begin{array}{l}\text { Mean } \mathrm{H}_{2} \text { particle density } \\
10^{4} \mathrm{~cm}^{-3}\end{array}$ \\
\hline \multicolumn{6}{|c|}{ observed in this paper: } \\
\hline Sgr C & $359: 45,-0.11$ & 1.7 & 2.5 & 7.9 & 1.8 \\
\hline $20 \mathrm{~km} \mathrm{~s}^{-1}$ & $359.87,-0.08$ & 5.1 & 33.9 & 17.6 & 0.9 \\
\hline $50 \mathrm{~km} \mathrm{~s}^{-1}$ & $359.98,-0.07$ & 2.7 & 6.5 & 7.2 & 1.2 \\
\hline $\mathrm{G} 0.253+0.016$ & $0.25,+0.02$ & 2.8 & 9.3 & 10.3 & 1.5 \\
\hline Sgr B1-off & $0.48,+0.00$ & 3.6 & 14.5 & 15.5 & 1.1 \\
\hline Sgr D & $1.13,-0.11$ & - & - & 3.4 & - \\
\hline \multicolumn{6}{|c|}{ ancillary CMZ clouds: } \\
\hline Dust Ridge C & $0.38,+0.04$ & - & - & 3.9 & - \\
\hline Dust Ridge D & $0.41,+0.05$ & 2.0 & 3.7 & 7.7 & 1.6 \\
\hline Sgr B2 & $0.66,-0.04$ & 7.1 & 136.5 & 101.8 & 1.3 \\
\hline
\end{tabular}

Notes. Radius and mass refer to the cloud area above an $\mathrm{H}_{2}$ column density of $9 \times 10^{22} \mathrm{~cm}^{-2}$, as derived from Herschel data in Sect. 2.4. The second to last column refers to the maximum mass per Herschel beam of $37^{\prime \prime}$ (i.e., $r_{\text {eff }}=0.74 \mathrm{pc}$ ) found within the aforementioned contour.

one target, the Sgr B2 complex, is removed from our sample for this reason. However, Paper II uses data from Schmiedeke et al. (2016) to fill in SMA-based density information on Sgr B2. In a final step we manually add the Sgr D region to this data set. This region was observed in the same setup used for other targets. However, the target resides slightly outside the region considered here.

We caution that Sgr D is likely to be a foreground or background object that is not physically associated with the CMZ. First, this region has relatively narrow lines (e.g., Mehringer et al. 1998; also see our results below). Second, Mehringer et al. (1998) demonstrate that broad absorption lines clearly associated with CMZ material are seen against the continuum of the Sgr D $\mathrm{H}$ II region. This suggests that the $\mathrm{H}$ II region resides behind the CMZ as discussed here. Third, Blum \& Damineli (1999) infer the presence of a molecular cloud extincting background stars at near-infrared wavelengths that must reside in front of the CMZ. Sawada et al. (2009) combine the evidence to conclude that the entire Sgr D complex consists of several physically unrelated components that are by chance seen along the same line of sight. For these reasons we follow a conservative approach when using data for this region. We present information for Sgr D as if located in the CMZ, but only as long as the region is easily identified in the tables and figures. Our conclusions are not influenced by this uncertainty.
This leaves us with a list of 6 targets. We here use observations of these regions that were collected using the SMA and the Atacama Pathfinder Experiment (APEX). Kauffmann et al. (2013b) already examined the SMA data for G0.253+0.016, and Kendrew et al. (2013) used some of the observations for the $\mathrm{Sgr} C$ region. The SMA continuum and $\mathrm{N}_{2} \mathrm{H}^{+}$data for the remaining clouds, plus the APEX data for all targets, have not previously been presented.

\subsection{SMA and APEX observations}

Interferometer Data We used the Submillimeter Array ( $\left.\mathrm{SMA}^{4}\right)$ to observe the $\mathrm{N}_{2} \mathrm{H}^{+}$(3-2) line and dust continuum near $280 \mathrm{GHz}$. An observing log is provided in Table 2. A total of four tracks was obtained on 2009 June 2 and $4\left(\approx 0.44 \mathrm{~km} \mathrm{~s}^{-1}\right.$ resolution, $4 \mathrm{GHz}$ combined total bandwidth) and 2012 May 29 and $30\left(\approx 0.87 \mathrm{~km} \mathrm{~s}^{-1}\right.$ resolution, $8 \mathrm{GHz}$ total bandwidth). Every target was mosaiced by observing several positions separated at less than half a $42^{\prime \prime}$ primary beam. The $345 \mathrm{GHz}$ receiver was tuned to the $\mathrm{N}_{2} \mathrm{H}^{+}$line in the LSB spectral band s4, using 256 channels per chunk and 24 chunks per sideband in 2009

4 The Submillimeter Array is a joint project between the Smithsonian Astrophysical Observatory and the Academia Sinica Institute of Astronomy and Astrophysics, and is funded by the Smithsonian Institution and the Academia Sinica. 
Table 2. Observing log for the SMA observations exploited in this paper.

\begin{tabular}{llll}
\hline \hline Date & Targets & Setup & Flux of 1744-312 \\
\hline 2009 June 2 & Sgr C, Sgr D & $4 \mathrm{GHz}^{a}, 0.44 \mathrm{~km} \mathrm{~s}^{-1}$ & $0.23 \mathrm{mJy}$ \\
2009 June 4 & G0.253+0.016 & $4 \mathrm{GHz}^{a}, 0.44 \mathrm{~km} \mathrm{~s}^{-1}$ & $0.28 \mathrm{mJy}$ \\
2012 May 29 & $50 \mathrm{~km} \mathrm{~s}^{-1}$, Sgr B1 Off & $8 \mathrm{GHz}^{b}, 0.87 \mathrm{~km} \mathrm{~s}^{-1}$ & $0.23 \mathrm{mJy}$ \\
2012 May 30 & $20 \mathrm{~km} \mathrm{~s}^{-1}$ & $8 \mathrm{GHz}^{b}, 0.87 \mathrm{~km} \mathrm{~s}^{-1}$ & $0.20 \mathrm{mJy}$ \\
\hline
\end{tabular}

Notes. ${ }^{(a)}$ Frequencies of 277.8 to $279.8 \mathrm{GHz}$ and 287.8 to $289.8 \mathrm{GHz}$; ${ }^{(b)}$ frequencies of 275.8 to $279.8 \mathrm{GHz}$ and 287.8 to $291.8 \mathrm{GHz}$.

June, respectively 128 channels per chunk and 24 chunks per sideband in 2012 May. This produces LSB frequency ranges of $v / \mathrm{GHz}=[277.8,279.8]$ in 2009 and $[275.8,279.8]$ in 2012 , and USB ranges of [287.8,289.8] and [287.8, 291.8], respectively. The bandpass calibrator was 3C 273 in 2009, while 3C 379 was used in 2012. Flux calibration is based on Titan in 2009, and on Uranus in 2012. Seven antennas were available in each observing session. They were arranged in the "compact north" and "compact" configurations in 2009 and 2012, respectively. Data were taken under good weather conditions with zenith optical depth $<0.1$ at $225 \mathrm{GHz}$ and single-sideband system temperatures $<200 \mathrm{~K}$. Further observations of G0.253+0.016 at about $219 \mathrm{GHz}$ from 2012 May were taken as part of this project, but these data are not relevant for the current publication.

The data were calibrated within MIR $^{5}$ using standard procedures. This involves flagging as well as the calibration of bandpass, flux, and phase. Consistency checks on the flux scale were done on 1744-312. The flux of this source varies on long timescales, but Table 2 shows that the flux calibration is consistent between consecutive observations.

The spectral data are further filtered using the UVLIN task from the MIRIAD ${ }^{6}$ package (Sault et al. 2006). Specifically, line-free channels are used to produce an averaged continuum signal for continuum imaging. The same channels are also used to estimate and subtract the continuum signal for later imaging of pure line emission.

Single-dish data. Complementary single-dish observations of the $\mathrm{N}_{2} \mathrm{H}^{+}$(3-2) line were obtained with the Atacama Pathfinder Experiment $\left(\mathrm{APEX}^{7}\right)$. Targets were observed using on-the-fly scans with $\leq 10^{\prime \prime}$ spacing between rows. The half-power beam width is 22 ".3 at the observed frequency, but smoothing during the on-the-fly data reduction increases the beam size to 23 !'7. Two or more perpendicular coverages were obtained to mitigate scanning stripes. Data were taken in 2014 on April 29, May 2, and May 7 under good weather conditions with system temperatures $\leq 180 \mathrm{~K}$. Data from the $\mathrm{FLASH}^{+}$receiver were recorded with the XFFTS backend at a velocity resolution of $0.04 \mathrm{~km} \mathrm{~s}^{-1}$. This setup produced data in the frequency ranges $v / \mathrm{GHz}=[280.0,282.5],[292.0,294.5]$, [450.1, 452.6], and $[463.6,466.1]$, but here we only exploit the information on the $\mathrm{N}_{2} \mathrm{H}^{+}$line. Data reduction includes standard calibration procedures and the subtraction of baselines of second order. The spectra are gridded to a final velocity resolution of $1.0 \mathrm{~km} \mathrm{~s}^{-1}$.

\footnotetext{
5 https://www.cfa.harvard.edu/sma/mir/

6 http://www .atnf.csiro.au/computing/software/miriad/

7 This publication is based on data acquired with the Atacama Pathfinder Experiment (APEX). APEX is a collaboration between the Max-Planck-Institut fur Radioastronomie, the European Southern Observatory, and the Onsala Space Observatory.
}

Line properties. Throughout this paper we adopt a rest frequency of $279511.8 \mathrm{MHz}$ for the $\mathrm{N}_{2} \mathrm{H}^{+}$(3-2) line (Caselli et al. $2002 \mathrm{~b})$. The hyperfine structure of the $\mathrm{N}_{2} \mathrm{H}^{+}$(3-2) line is unlikely to have a significant impact on the observed line structure. A compact summary is, for example, provided by Table A.1 of Caselli et al. (2002b). This demonstrates that hyperfine satellites with velocity offsets exceeding $\pm 0.6 \mathrm{~km} \mathrm{~s}^{-1}$ only contain a few percent of the integrated relative intensities. In summary, satellites within $\pm 0.6 \mathrm{~km} \mathrm{~s}^{-1}$ offset from the line reference frequency might broaden the observed lines by about $1 \mathrm{~km} \mathrm{~s}^{-1}$, but the hyperfine structure is unlikely to have further impact on the observed spectra.

The $\mathrm{N}_{2} \mathrm{H}^{+}$(3-2) transition has an upper-state energy of $E_{\mathrm{u}} / k_{\mathrm{B}}=26.8 \mathrm{~K}$, and an Einstein- $A_{i j}$ coefficient of $1.26 \times 10^{-3} \mathrm{~s}^{-1}$ (Müller et al. 2001, 2005). This yields critical densities of order $10^{6} \mathrm{~cm}^{-3}$ under typical CMZ conditions with gas temperatures $\lesssim 100 \mathrm{~K}$ (Shirley 2015). $\mathrm{N}_{2} \mathrm{H}^{+}$is the main workhorse tracer of dense gas in molecular clouds with typical abundances $\sim 10^{-10}$ (e.g., Tafalla et al. 2006). The molecule is believed to be primarily a tracer of gas that is cold enough for depletion of $\mathrm{CO}$ on dust since $\mathrm{N}_{2} \mathrm{H}^{+}$is presumably destroyed by $\mathrm{CO}$ (Caselli et al. 2002a).

\subsection{Joint imaging of data}

The interferometer and single-dish data are combined to produce maps that contain information on both small and large angular scales. We use separate processes for the continuum and emission line data. Very few resources provide a hands-on introduction on the combination of interferometer and single-dish data. We therefore continue to collect and document procedures at

\section{http://tinyurl.com/zero-spacing}

Other relevant discussions of this problem are provided by Zhang et al. (2000), Kurono et al. (2009), and the IRAM technical report ${ }^{8} 2008-2$ by Rodriguez-Fernandez, Pety \& Gueth.

We apply a "primary beam correction" to all interferometer maps analyzed here, in order to remove spatial gain variations in the images, unless noted otherwise. The maps shown here do not include this correction, though, since this suppressed noise at map boundaries.

We use the Common Astronomy Software Applications package $\left(\mathrm{CASA}^{9}\right)$ to image the dust continuum emission. We build on the procedures of Zhang et al. (2000). Details of the processing are provided in Appendix A.1. First, the interferometer observations are imaged and cleaned. We improve the performance by providing an iteratively improved source model to CASA's CLEAN procedure via the "modelimage" option. We also use the "multiscale" option to suppress artifacts from mildly extended

\footnotetext{
8 Accessible at http://www.iram-institute.org

9 http://casa.nrao.edu/
} 


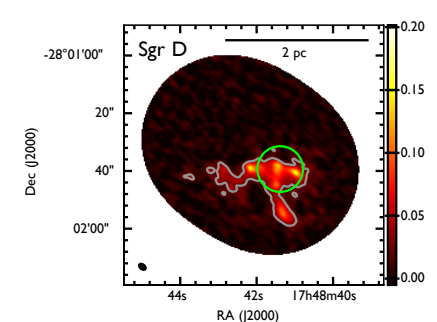

intensities given in Jy beam ${ }^{-1}$

$\mathrm{H}_{2}$ column density range in color scale: 0 to $1.2 \times 10^{24} \mathrm{~cm}^{-2}$

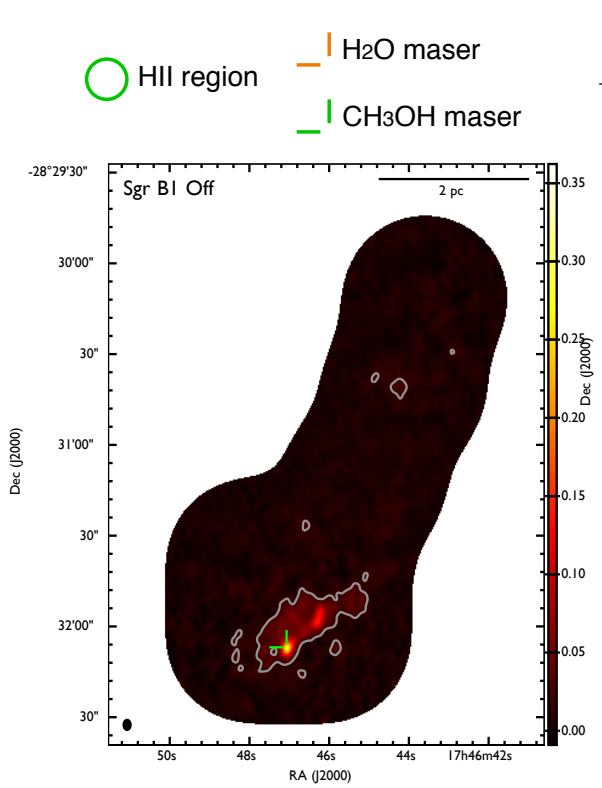

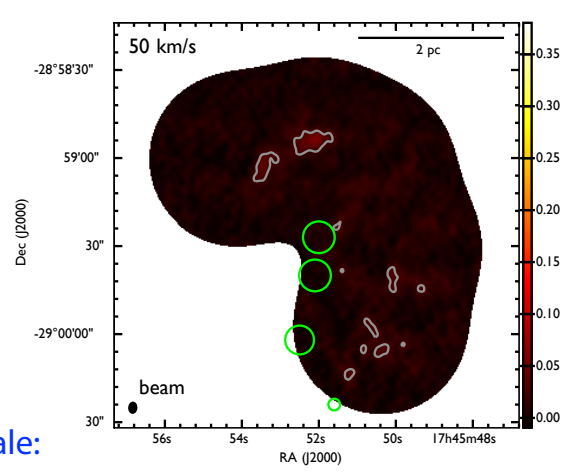

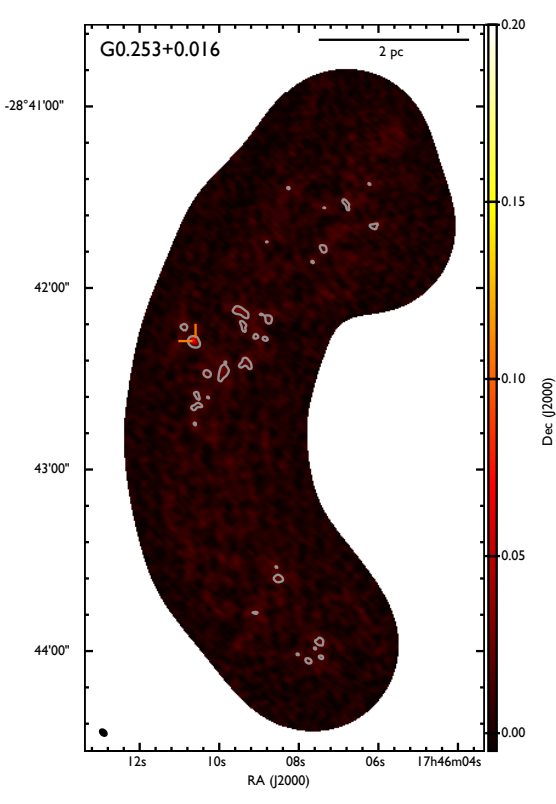

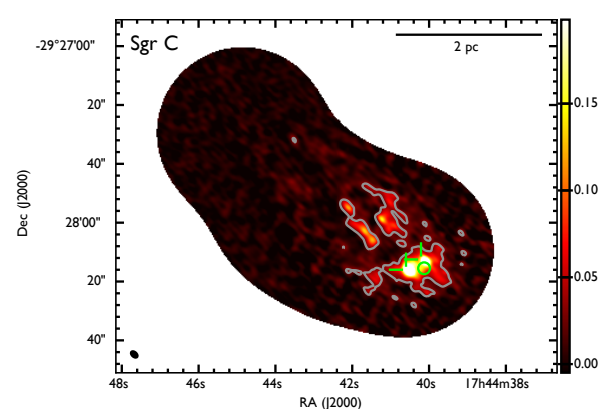

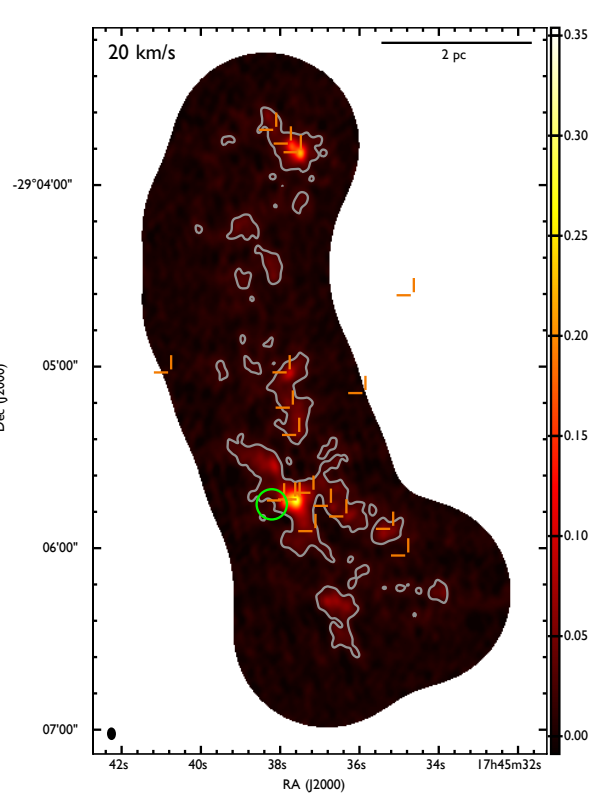

Fig. 2. Maps of dust continuum emission produced from the SMA interferometer data alone. Intensities are multiplied by gain distributions that suppress noise at the map boundaries. Beam shapes and sizes are indicated in the lower left corner of each panel, while a scale bar in the top right corner indicates physical dimensions. Color bars labeled in units of $\mathrm{Jy} \mathrm{beam}^{-1}$ give the intensity scale separately for every image. We note that the beam size varies between maps. We choose the color bar to represent a fixed $\mathrm{H}_{2}$ column density range of $[0,1.2] \times 10^{24} \mathrm{~cm}^{-2}$. In other words, images are represented in a manner that makes column densities directly comparable. A solid contour indicates the observed intensity exceeds the noise by a factor 5 . Overlaid green circles, green crosses, and orange crosses indicate the locations of H II regions, methanol masers, and water masers, respectively.

emission. Second, the interferometer images are combined with single-dish estimates of dust continuum. The latter data are generated by scaling observations from the APEX Telescope Large Area Survey of the Galaxy (ATLASGAL, Schuller et al. 2009; see Contreras et al. 2013 for data reduction details) that were done at a wavelength of $870 \mu \mathrm{m}$ using the Large APEX Bolometer Camera (LABOCA; Siringo et al. 2007, 2009) to the observing frequency of the SMA. See Appendix A.1 for details. We choose ATLASGAL data over the data provided by Bolocam (Aguirre et al. 2011; Bally et al. 2010) because of the higher angular resolution. The FEATHER algorithm from CASA is used to execute this combination. We stress that the data combination via FEATHER is an approximate procedure that assumes perfect sampling of the $u v$-plane out to the largest baselines observed by the interferometers. Where possible we therefore rely on the interferometer-only imaging products.

Figure 2 presents the maps of dust continuum emission for this study. Indicators of star formation are overlaid following the information compiled in Appendix B. Noise levels and beam shapes are summarized in Table 3. We note that Fig. 2 shows maps without single-dish data folded in. The data are also not corrected for the aforementioned gain distributions. This suppresses signal but also noise at the map boundaries. We choose this representation in order to reflect the quality of the interferometer data. The intensities in Fig. 2 are scaled to highlight a fixed column density range for all clouds. The conversion between dust intensities and column densities follows Appendix A from Kauffmann et al. (2008). We adopt dust temperatures of $20 \mathrm{~K}$ (see Sect. 2.4) and Ossenkopf \& Henning (1994) dust opacities for thin ice mantles that have coagulated at a density of $10^{6} \mathrm{~cm}^{-3}$ for $10^{5} \mathrm{yr}$, as approximated by Battersby et al. (2011), that are reduced by a factor 1.5 to be consistent with our previous work (see Kauffmann et al. 2010).

The maps are largely devoid of significant emission. This is a main feature of G0.253+0.016 already reported by Kauffmann et al. (2013b). The new observations now show that this relative absence of bright continuum emission on small spatial scales is a general feature of CMZ molecular clouds. We defer a detailed discussion of this trend to Paper II that is focused on the cloud density structure. In that publication we demonstrate that $\lesssim 10 \%$ of the total cloud mass typically resides in structures detected by the SMA. 
Table 3. Summary of image properties.

\begin{tabular}{|c|c|c|c|c|c|}
\hline Target & \multicolumn{2}{|c|}{ Setup } & Noise & Beam size & Beam position angle \\
\hline \multirow[t]{2}{*}{ Sgr C } & $4 \mathrm{GHz}$ & cont. & $6.5 \mathrm{mJy}$ beam $^{-1}$ & $2 \prime \prime 6 \times 11^{\prime \prime} 6$ & $48^{\circ}$ \\
\hline & & $\mathrm{N}_{2} \mathrm{H}^{+}$ & $39 \mathrm{mK}$ & $5^{\prime \prime} .0 \times 4^{\prime \prime} .7$ & $124^{\circ}$ \\
\hline \multirow[t]{2}{*}{$20 \mathrm{~km} \mathrm{~s}^{-1}$} & $8 \mathrm{GHz}$ & cont. & $3.9 \mathrm{mJy}$ beam $^{-1}$ & $3 \prime \prime 4 \times 2 ! ! 2$ & $2^{\circ}$ \\
\hline & & $\mathrm{N}_{2} \mathrm{H}^{+}$ & $36 \mathrm{mK}$ & $6^{\prime \prime} .0 \times 5^{\prime \prime} .2$ & $162^{\circ}$ \\
\hline \multirow[t]{2}{*}{$50 \mathrm{~km} \mathrm{~s}^{-1}$} & $8 \mathrm{GHz}$ & cont. & $4.6 \mathrm{mJy}$ beam $^{-1}$ & $3 \prime .4 \times 2 ! .4$ & $177^{\circ}$ \\
\hline & & $\mathrm{N}_{2} \mathrm{H}^{+}$ & $58 \mathrm{mK}$ & $5 \prime .6 \times 4 " .8$ & $159^{\circ}$ \\
\hline \multirow[t]{2}{*}{$\mathrm{G} 0.253+0.016$} & $4 \mathrm{GHz}$ & cont. & $3.5 \mathrm{mJy}$ beam $^{-1}$ & $2^{\prime \prime} .5 \times 1^{\prime \prime} .7$ & $48^{\circ}$ \\
\hline & & $\mathrm{N}_{2} \mathrm{H}^{+}$ & $42 \mathrm{mK}$ & $6 \prime .7 \times 5^{\prime \prime} .9$ & $116^{\circ}$ \\
\hline \multirow[t]{2}{*}{ Sgr B1-off } & $8 \mathrm{GHz}$ & cont. & $6.8 \mathrm{mJy}$ beam $^{-1}$ & $3 \prime \prime 3 \times 2 ! \prime 3$ & $0^{\circ}$ \\
\hline & & $\mathrm{N}_{2} \mathrm{H}^{+}$ & $63 \mathrm{mK}$ & $5^{\prime \prime} .4 \times 4^{\prime \prime} .8$ & $163^{\circ}$ \\
\hline \multirow[t]{2}{*}{ Sgr D } & $4 \mathrm{GHz}$ & cont. & $5.5 \mathrm{mJy}$ beam $^{-1}$ & $2 \prime \prime 6 \times 1 " .6$ & $50^{\circ}$ \\
\hline & & $\mathrm{N}_{2} \mathrm{H}^{+}$ & $75 \mathrm{mK}$ & $3 \prime \prime 7 \times 3 \prime \prime 6$ & $42^{\circ}$ \\
\hline
\end{tabular}

Notes. The noise level for spectral line observations is determined for channels of $1 \mathrm{~km} \mathrm{~s}^{-1}$ width. The bandwidth for continuum observations depends on the observing season, as indicated in Table 2. The position angle of the beam is measured east of north.

We jointly image interferometer and single-dish $\mathrm{N}_{2} \mathrm{H}^{+}$line emission data to recover both compact and extended emission. To do this we start by combining the data in the $u v$-domain. This requires a complex pipeline that is implemented using MIRIAD and further explained in Appendix A.2. In brief, we process the single-dish maps to generate artificial data points in the $u v$-plane that represent emission on large spatial scales. We then combine these data with the regular $u v$-plane data points from the interferometer. Weighting functions are used to produce a blended data set that represents emission on both small and large scales. These combined data are then imaged and cleaned to produce a final image. This detailed combination of the single-dish and interferometer data produces a larger restoring beam than that obtained for interferometer-only data (i.e., in dust continuum).

This procedure produces good maps of all GCMS targets, except for selected regions and velocity channels in the $20 \mathrm{~km} \mathrm{~s}^{-1}$ cloud. Specifically we obtain maps containing strong "corrugations" in some northern parts of this cloud. We attribute this to bright $\mathrm{N}_{2} \mathrm{H}^{+}$emission outside of the region mapped by the SMA. In this case too much information is missing to properly interpret the signal picked up by the interferometer. The properties quoted for the $20 \mathrm{~km} \mathrm{~s}^{-1}$ cloud might be biased due to these problems, in particular if measurements are made for compact objects. But we do not think that these problems influence the conclusions obtained in this paper. We note that the dust continuum emission is well covered by our maps and consequently these data do not suffer from imaging artifacts.

\subsection{Dust observations with Herschel}

We use data from the Herschel Space Telescope to provide a context on cloud density structure within which the interferometer data obtained here can be interpreted. Dust emission images of the region $|\ell| \lesssim 2^{\circ}$ are used for this purpose. We also use Herschel observations to obtain similar data on the cloud density structure of the Orion A molecular. This information is used to obtain reference data against which the structure of CMZ clouds can be explored. The Orion data are primarily exploited in Paper II. The processing of these maps is described here to provide a clear picture of Herschel data reduction principles.

We use public Herschel data observed as part of the Herschel Infrared Galactic Plane Survey (Hi-GAL, Molinari et al. 2010) and the Gould Belt Survey (GBS, André et al. 2010)
Table 4. Summary of the Herschel dust continuum observations used in this study.

\begin{tabular}{cccc}
\hline \hline Target name & Observation IDs & Obs. date & Project \\
\hline Field-000_0 & $1342204102 / 3$ & $2010-09-07$ & Hi-GAL \\
Field-002_0 & $1342204104 / 5$ & $2010-09-07$ & Hi-GAL \\
Field-004_0 & $1342214761 / 2$ & $2011-02-24$ & Hi-GAL \\
OrionA-N-1 & $1342218967 / 8$ & $2011-04-09$ & GBS \\
OrionA-C-1 & $1342204098 / 9$ & $2010-09-06$ & GBS \\
OrionA-S-1 & $1342205076 / 7$ & $2010-09-26$ & GBS \\
\hline
\end{tabular}

key projects. Hi-GAL and the GBS cover, among other targets, the CMZ and the Orion molecular cloud, respectively. Table 4 indicates the target name, the observation IDs, observing dates, and the associated projects of the data used in this work. All these observations were taken using the parallel mode in which five wavebands $(70,160,250,350$, and $500 \mu \mathrm{m})$ are observed simultaneously. For the CMZ field we also include maps from the ATLASGAL project on APEX (Schuller et al. 2009; Contreras et al. 2013). These long-wavelength data cannot be included in the Orion analysis due to strong spatial filtering of the bolometer observations.

Herschel data for the CMZ are reduced and processed following the procedure described in Guzmán et al. (2015). Mapping and destriping are done using standard tools provided by the Herschel Interactive Processing Environment (HIPE) version 10. Maps are convolved to the resolution of the $500 \mu \mathrm{m}$ maps (i.e., 37") by using ad-hoc convolution kernels (Aniano et al. 2012) and transformed to a common pixel grid. The procedure described in Guzmán et al. (2015) includes the subtraction of a smooth component representing diffuse emission from the Galactic Plane. We refer to this diffuse component as a "background", bearing in mind that it could equally represent background or foreground emission. The objective of this background subtraction is twofold: (i) to match the subtraction of the low spatial frequency signal that affects the ATLASGAL data; and (ii) to subtract diffuse emission that cannot be attributed to the individual CMZ clouds that are the targets of this study. As an example, we show the performance of the background subtraction around the $20 \mathrm{~km} \mathrm{~s}^{-1}$ molecular cloud. This cloud is located very near the Galactic center, where significant diffuse emission is present. Figure 3 shows the $350 \mu \mathrm{m}$ intensity measured by 
Herschel versus the Galactic latitude across the approximate longitude of the $20 \mathrm{~km} \mathrm{~s}^{-1}$ molecular cloud $(\ell=359: 87)$. It is apparent that the subtracted background is not affecting the molecular cloud emission, and conversely, not subtracting this component could induce one to overestimate the column density of the CMZ clouds (or of any molecular cloud located in the Galactic plane). Moreover, the background subtraction also corrects for the unknown offset of the Herschel photometry which is shifted by an unknown quantity independent for each field.

Background-subtracted images are combined and a single temperature dust emission model is fitted to the intensities from 160 to $870 \mu \mathrm{m}$ for each pixel. The main difference between the procedure employed in this work and the one described in Guzmán et al. (2015) is that we base our assumed description of the dust opacity prescription on the properties listed by Ossenkopf \& Henning (1994). Here we assume thin ice coatings and dust coagulation for $10^{5} \mathrm{yr}$ at a molecular volume density of $10^{6} \mathrm{~cm}^{-3}$, and we further decrease the resulting opacity by a factor 1.5. This approach is chosen to be consistent with our previous work on dust-based mass estimates (see Kauffmann et al. 2010). We note that our masses are slightly lower than those Walker et al. (2015) quote for the same regions. The reason for this remains unclear, in particular given that the lower dust opacities assumed by us should yield higher masses for given flux.

Data for the Orion A molecular cloud are reduced in a similar way. These data are not exploited in the current publication, but they play a major role as a reference data set in other GCMS publications. We note that we confine the analysis to wavelengths between 250 and $500 \mu \mathrm{m}$. We do so because visual inspection suggests that some of the emission at lower wavelengths is not associated with dense gas. For example, due to excitation from the OB-type stars in the Orion Nebula, a significant fraction of the intensity might be due to emission from very small grains that are not tracing the dense interior of the cloud (Schnee et al. 2008). The calibrated data sets are obtained from the Herschel Science Archive (Standard Product Generation version 9.2.0). The mapping, destriping, and combination of the three fields is carried out using Unimap (Piazzo et al. 2015). As done for the CMZ, we also subtract a background in order to distinguish Galactic plane diffuse emission from the molecular cloud (and simultaneously correct for the photometric offset). For Orion A, however, we consider a much smoother background compared to those subtracted to the $\mathrm{CMZ}$ fields. While the CMZ background filters out uniform emission on scales of $\sim 2.5$, the Orion $A$ background subtraction filters out uniform emission on scales of $\sim 15^{\prime}$. The Orion A far-IR Herschel maps are convolved, regridded, and column density and temperature maps are fitted to the intensities in a similar fashion as done with the CMZ. The Herschel data for Orion have previously for example been imaged by Lombardi et al. (2014) using alternative data processing algorithms.

We use the Herschel data to obtain two sets of mass measurements for every CMZ cloud. First, we characterize a large column density contour that still clearly selects the target cloud. In practice we extract all clouds at an $\mathrm{H}_{2}$ column density of $9 \times 10^{22} \mathrm{~cm}^{-2}$ to do this. For this contour we obtain the enclosed mass and the effective radius, $r_{\text {eff }}=(A / \pi)^{1 / 2}$, from the enclosed area, $A$. Second, within this contour we determine for every target cloud the maximum mass contained in a Herschel beam of $37^{\prime \prime}$, corresponding to $r_{\text {eff }}=0.74$ pc. Results are collected in Table 1.

We obtain dust temperatures in the range 17 to $25 \mathrm{~K}$ for our target clouds. This is consistent with previously reported Herschel-based temperatures (Longmore et al. 2012). Assuming

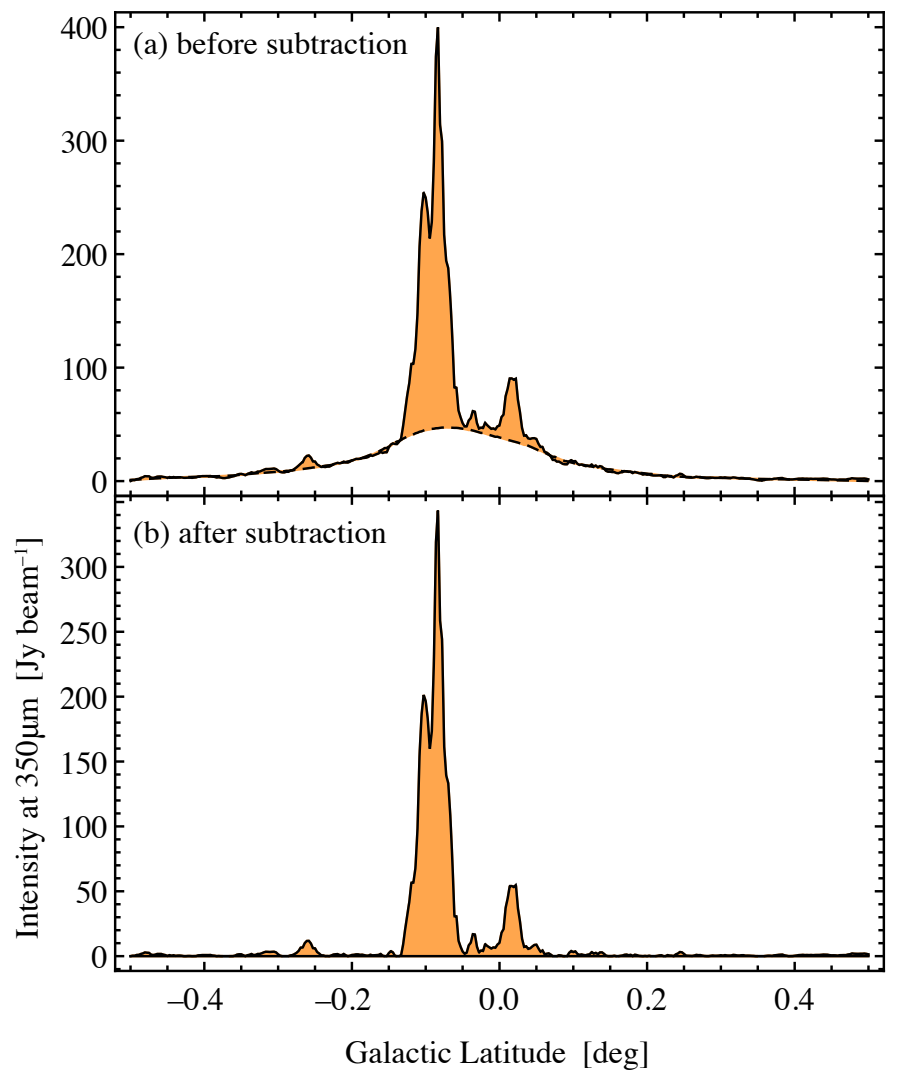

Fig. 3. Example of the background subtraction at $\ell=3599^{\circ} 87$. The most conspicuous feature corresponds to the $20 \mathrm{~km} \mathrm{~s}^{-1}$ cloud.

a spherical geometry, uniform density, and a mean molecular weight per $\mathrm{H}_{2}$ molecule of 2.8 proton masses (Kauffmann et al. 2008), we evaluate the mean cloud densities as $n\left(\mathrm{H}_{2}\right)=3.5 \times$ $10^{4} \mathrm{~cm}^{-3} \cdot\left(M / 10^{4} M_{\odot}\right) \cdot(r / p c)^{-3}$. The cloud masses and radii reported in Table 1 give average densities of $(0.9$ to 1.8$) \times 10^{4} \mathrm{~cm}^{-3}$ for the target clouds.

\section{Narrow lines and linewidth-size relation}

A major surprise of the Kauffmann et al. (2013b) study of G0.253+0.016 was the detection of very narrow lines with widths $\leq 1 \mathrm{~km} \mathrm{~s}^{-1}$ in structures with spatial scales $\lesssim 0.1 \mathrm{pc}$. This is remarkable given the strong unordered gas motions of $\geq 10 \mathrm{~km} \mathrm{~s}^{-1}$ on the largest spatial scales (e.g., Lis et al. 1994).

Here we take a thorough look at the single-dish and interferometer data to develop a comprehensive view of $\mathrm{CMZ}$ cloud kinematics. This work employs the well-detected $\mathrm{N}_{2} \mathrm{H}^{+}(3-2)$ transition. Here we use the combined data of APEX and the SMA for our analysis. We find that the results of Kauffmann et al. (2013b) are not affected by spatial filtering and are representative of the entire $\mathrm{CMZ}$.

\subsection{Properties of $\mathrm{N}_{2} \mathrm{H}^{+}$maps}

The $\mathrm{N}_{2} \mathrm{H}^{+}$maps are very rich in kinematic structure. Figures 4 and 5 give an impression of the complexity found in the maps, while Table 3 summarizes aspects of the image quality.

Panel a of Fig. 4 zooms in onto the northern end of $\mathrm{G} 0.253+0.016$. We note the significant variations between velocity channels. This is remarkable, given that the channels have a width of only $1 \mathrm{~km} \mathrm{~s}^{-1}$. Panels $\mathrm{b}$ and c present spectra for two of 


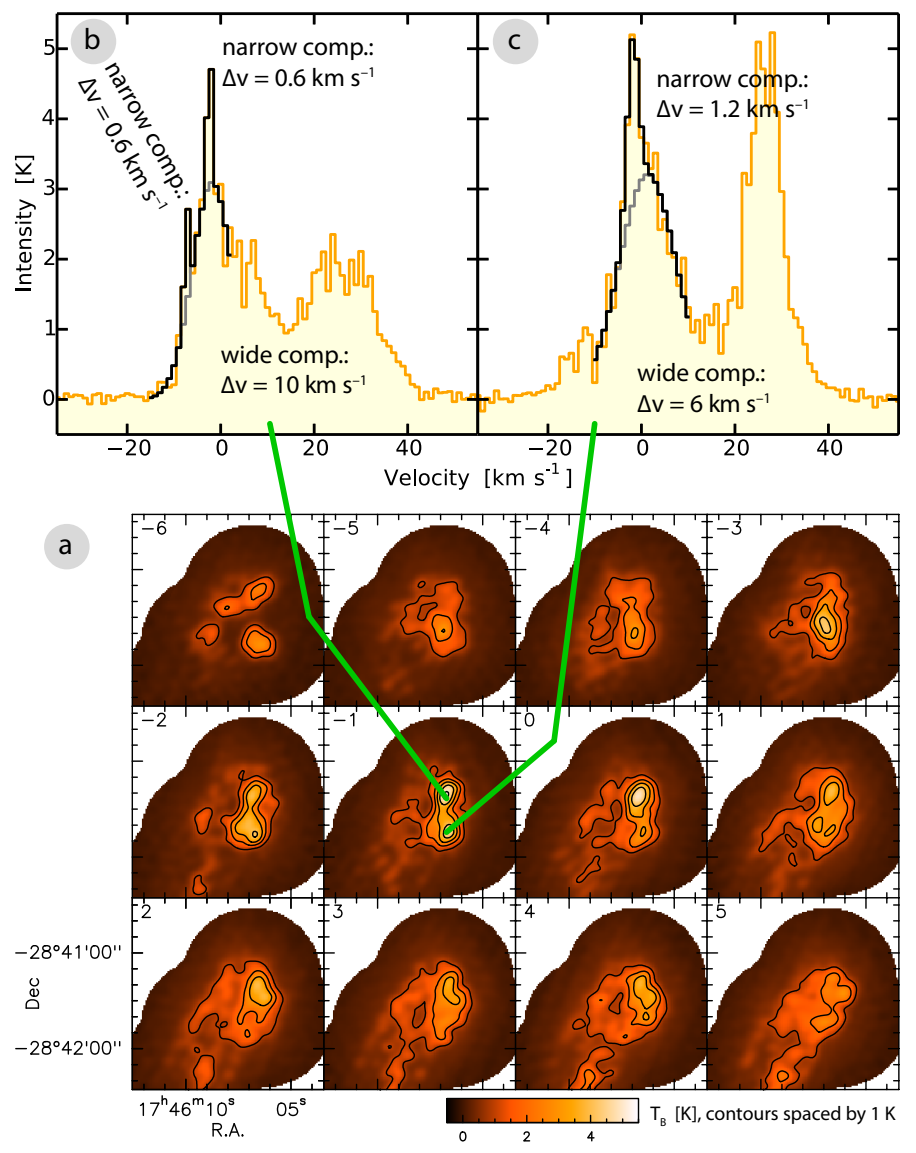

Fig. 4. $\mathrm{N}_{2} \mathrm{H}^{+}$(3-2) data for a sample region in the north of G0.253+0.016. Panel a) presents channel maps. The velocity in $\mathrm{km} \mathrm{s}^{-1}$ is indicated in the upper left corner. Panels b) and c) show spectra extracted towards selected positions. Black lines in these plots depict multi-component Gaussian fits to selected velocity ranges of the spectra, where gray lines trace the structure of the widest component. For each component we indicate the full width at half maximum, $\Delta v$.

the intensity peaks seen in the map. In combination these panels reveal that features of very narrow line width, sometimes comparable to the width of individual velocity channels, are superimposed on structures with much higher line width. This is explicitly demonstrated via simultaneous Gaussian fits to parts of the spectra in Fig. 4. Specifically, the spectra reveal two features with line widths that are too small to be properly resolved by our channels of $1 \mathrm{~km} \mathrm{~s}^{-1}$ spacing. But they also expose several additional velocity components, including features with widths of 6 to $10 \mathrm{~km} \mathrm{~s}^{-1}$ on which the more narrow lines are superimposed.

Figure 5, presenting data towards the southern part of G0.253+0.016, reveals a qualitatively different structure in the kinematic information. Here we find a much more complex spectrum with multiple velocity components of relatively narrow width crowding within a velocity range of about 20 to $30 \mathrm{~km} \mathrm{~s}^{-1}$ width. We also note the difference in bulk velocity compared to Fig. 4.

Data of the sort depicted in Figs. 4 and 5 reveal three broad trends in cloud structure: (i) the existence of very narrow components that are barely resolved by our channels with $1 \mathrm{~km} \mathrm{~s}^{-1}$ spacing; (ii) the presence of broader velocity components with a width $\lesssim 10 \mathrm{~km} \mathrm{~s}^{-1}$ (which might well be superpositions of several unresolved components); and (iii) the fact that several wellseparated relatively wide velocity components can exist along the same line-of-sight. The latter trend is for example evident in

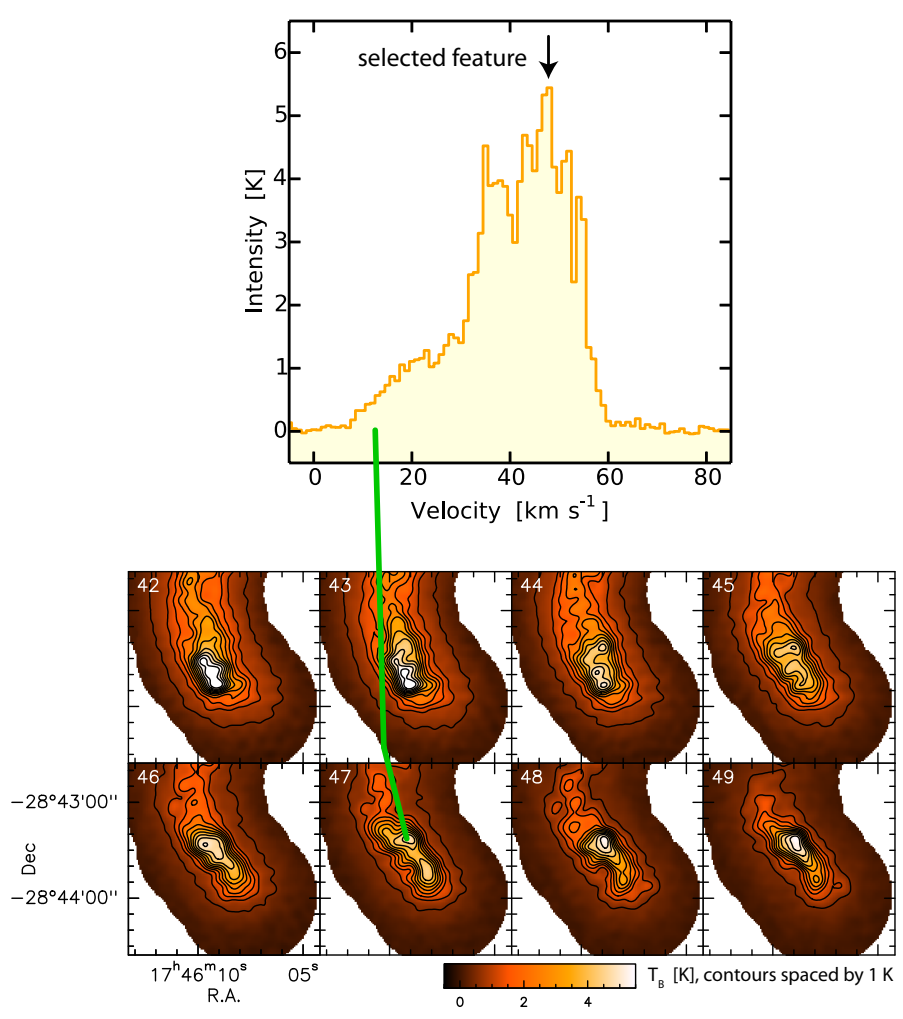

Fig. 5. As Fig. 4, but for a southern section of G0.253+0.016. Map and spectrum indicate a feature selected in the dendrogram-based search for compact features in the $\mathrm{N}_{2} \mathrm{H}^{+}$data.

the northern part of G0.253+0.016 (Fig. 4c), where two broad features separated by $\sim 30 \mathrm{~km} \mathrm{~s}^{-1}$ with peak intensities $\sim 5 \mathrm{~K}$ are evident.

The images also reveal a low level of imaging artifacts in the form of corrugations. The maximum amplitude of these artifacts is estimated manually by inspecting the depth of the minima between consecutive peaks that appear to follow the corrugation pattern. This yields a very conservative upper limit of $\leq 1 \mathrm{~K}$ for the imaging artifacts. As mentioned before, these artifacts are larger for the northern part of the $20 \mathrm{~km} \mathrm{~s}^{-1}$ cloud where bright emission outside the map prevents a clean image reconstruction (Sect. 2.3).

The comparison of the $\mathrm{N}_{2} \mathrm{H}^{+}$and dust emission maps reveals a surprising trend: there is no close correspondence between the two on the angular scales probed by the interferometer. Some of the dust emission peaks seen at high angular resolution are also seen as peaks in $\mathrm{N}_{2} \mathrm{H}^{+}$channel maps, but sometimes this requires close inspection of the data, and bright $\mathrm{N}_{2} \mathrm{H}^{+}$features usually have no obvious counterparts in dust emission. Better agreement is found on larger angular scales probed by singledish data (e.g., Rathborne et al. 2014a).

Similar trends have, for example, been reported by Rathborne et al. (2015). That study focusses on lines in the $3 \mathrm{~mm}$ band. Many of those emission lines are optically thick, and Rathborne et al. (2015) argue that radiative transfer effects could in part explain the lack of correlation between the line and dust data. However, such an explanation seems unlikely for the $\mathrm{N}_{2} \mathrm{H}^{+}$ (3-2) lines explored here, which have higher excitation (i.e., temperature and density) requirements and should have a much lower optical depth. This leaves complex spatial variations in the chemical abundance as another explanation for the disconnect between maps of dust and line emission, as also suggested 
by Rathborne et al. (2015). Further, recall that the CMZ gas temperatures of 50 to $100 \mathrm{~K}$ are distinct from the dust temperatures $\sim 20 \mathrm{~K}$. These temperature differences between gas and dust can also help to reduce the correlation between molecular emission lines and dust emission.

\subsection{Segmentation of $\mathrm{N}_{2} \mathrm{H}^{+}$emission}

The complexity of the gas kinematics complicates the extraction of cloud fragments. We therefore follow a mixed approach.

In one scheme we start by finding the maximum intensity for a given cloud in position-position-velocity $(p-p-v)$ space. We then draw an iso-intensity surface in $p-p-v$ space at a threshold intensity corresponding to $1 / 3$ of this peak intensity. Contiguous regions within these surfaces are considered to be coherent structures. We refer to these structures as "clumps". This search and the object characterization are done using the ASTRODEN$\mathrm{DRO}^{10}$ implementation of the dendrogram segmentation algorithm described by Rosolowsky et al. (2008). The min_value parameter is used to reject regions below $1 / 3$ of the peak intensity. Our exploration below considers structures on all spatial scales and is thus robust with respect to the exact choice for the threshold intensity. The size of clumps is characterized via the effective radius $r_{\text {eff }}=(A / \pi)^{1 / 2}$, where $A$ is the area on the sky that contains all volume elements belonging to an extracted structure. Spectra integrated within the $p-p-v$ surfaces are used to obtain bulk velocities and velocity dispersions. The latter property is only calculated if the peak intensity of a clump exceeds the threshold intensity for clump selection by a factor of two (i.e., achieves $\geq 2 / 3$ of the peak intensity in the map). This restriction guarantees spectra with well-defined peaks and line wings that can be characterized meaningfully by a velocity dispersion. The detailed properties of extracted clumps are given in Table 5. We include measures of the spatial extent along the overall major and minor axes of the structures following Rosolowsky et al. (2008; see their Eq. (2)) and the position angle (PA) of the major axis measured east from north. The number of extracted clumps is listed in Table 6. With the notable exception of G0.253+0.016, all clouds have a rather simple structure that consists of only one or two clumps.

In a second scheme we use the single-dish data to obtain and characterize integrated spectra for the clouds. These are shown in Fig. 6. Table 6 lists the mean velocities, $\langle v\rangle_{\mathrm{SD}}$, and velocity dispersions, $\sigma_{\mathrm{SD}}(v)$, obtained as the first and second moment of these spectra.

In a third scheme we focus on the most narrow lines in regions at the resolution limit of our maps. This is described in the next section.

\subsection{Narrow lines in CMZ clouds}

We return to the quest for narrow lines in CMZ clouds, as initiated in Kauffmann et al. (2013b). A particular issue is the systematic characterization of the narrow lines, given the complex kinematics described in Sect. 3.1. These narrow components are in particular found towards prominent line emission peaks seen at the highest angular resolutions.

In our search for such features we first find all significant local maxima in $p-p-v$ space that are above the noise by a factor $\geq 5$. We also require that these maxima are separated from other significant maxima by troughs with a minimum depth larger than the amplitude of imaging artifacts (i.e., $1 \mathrm{~K}$ ). In practice these

\footnotetext{
10 http : //www . dendrograms . org
}

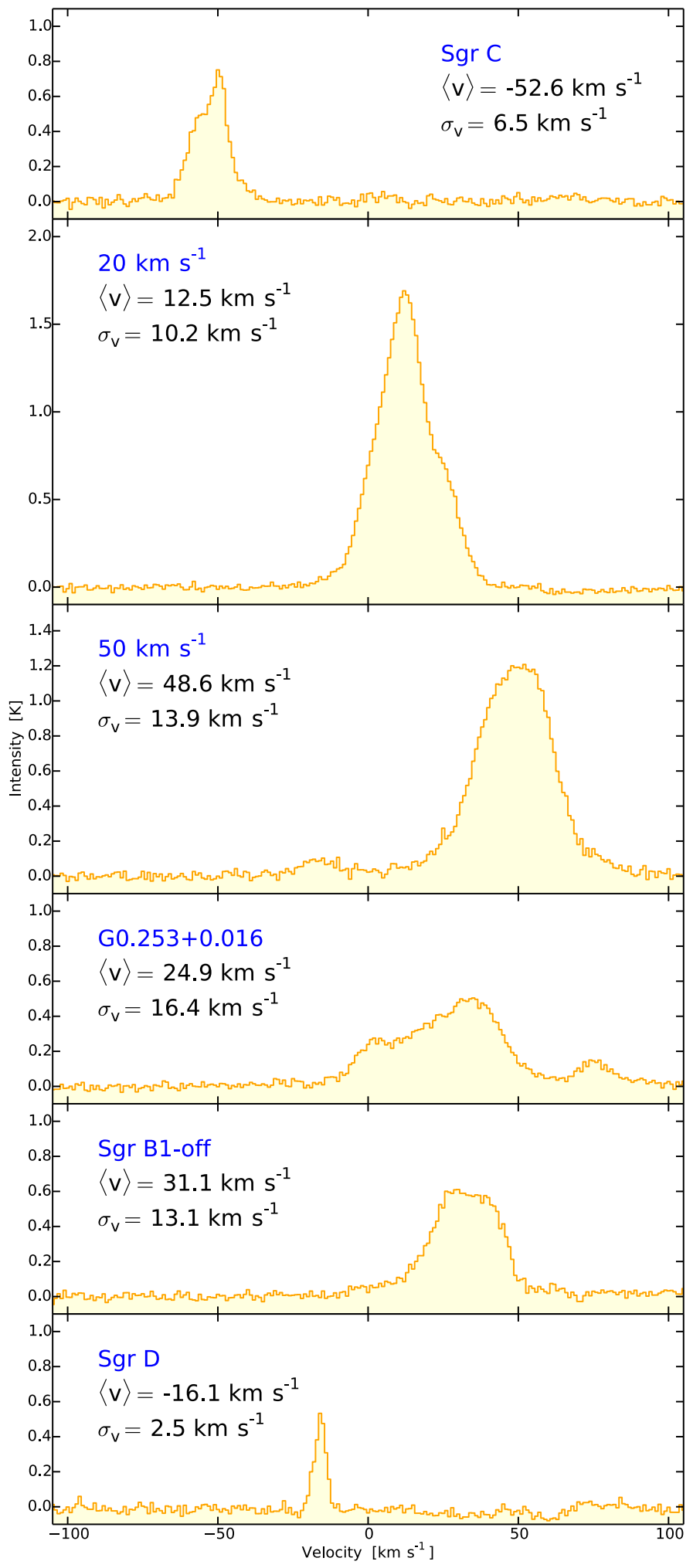

Fig. 6. Spatially averaged single-dish spectra of the $\mathrm{N}_{2} \mathrm{H}^{+}(3-2)$ transition for the target clouds. In each panel we indicate the mean velocity and velocity dispersion that are derived as the first and second moment of the spectra. See Sects. 3.2 and 3.3 for details.

criteria are implemented via the min_value and min_delta parameters in the ASTRODENDRO code. The extracted cloud fragments are not well separated from the general cloud emission. For this reason we do not attempt a characterization in terms of size and line width for all these features. Instead we 
Table 5. Properties of dendrogram-extracted clumps as described in Sect. 3.2.

\begin{tabular}{|c|c|c|c|c|c|c|}
\hline Region & \multicolumn{2}{|c|}{$\mathrm{J} 2000.0$} & $\begin{array}{l}r_{\mathrm{eff}} \\
\mathrm{pc}\end{array}$ & $\begin{array}{c}\text { Size @ PA } \\
\operatorname{arcsec} @ \text { deg }\end{array}$ & $\begin{array}{l}\langle v\rangle \\
\mathrm{km} \mathrm{s}^{-1}\end{array}$ & $\begin{array}{l}\sigma_{v} \\
\mathrm{~km} \mathrm{~s}^{-1}\end{array}$ \\
\hline Sgr C & $17: 44: 41.1$ & $-29: 28: 09.3$ & 0.7 & $9.1 \times 5.4 @ 41$ & -52.1 & 3.0 \\
\hline $20 \mathrm{~km} \mathrm{~s}^{-1}$ & $17: 45: 37.9$ & $-29: 04: 52.9$ & 1.8 & $56.9 \times 10.3 @ 14$ & 13.6 & 6.9 \\
\hline $50 \mathrm{~km} \mathrm{~s}^{-1}$ & $17: 45: 51.0$ & $-28: 59: 21.8$ & 1.2 & $24.5 \times 10.3 @ 24$ & 44.3 & 6.4 \\
\hline \multirow{5}{*}{$\mathrm{G} 0.253+0.016$} & $17: 46: 06.7$ & $-28: 41: 31.3$ & 0.6 & $9.8 \times 4.5 @-8$ & 0.6 & 3.1 \\
\hline & $17: 46: 07.2$ & $-28: 41: 26.9$ & 0.8 & $8.5 \times 8.1 @ 33$ & 27.5 & 4.1 \\
\hline & $17: 46: 09.4$ & $-28: 43: 18.1$ & 1.3 & $25.9 \times 8.8 @ 12$ & 40.8 & 5.9 \\
\hline & $17: 46: 09.7$ & $-28: 42: 32.4$ & 0.5 & $6.8 \times 4.1 @-35$ & 12.0 & 2.6 \\
\hline & $17: 46: 10.1$ & $-28: 43: 01.7$ & 0.4 & $5.3 \times 3.4 @ 9$ & 20.9 & 1.2 \\
\hline \multirow[t]{2}{*}{ Sgr B1 } & $17: 46: 43.5$ & $-28: 30: 14.0$ & 0.4 & $4.1 × 3.8 @ 57$ & 34.1 & 1.2 \\
\hline & $17: 46: 46.8$ & $-28: 31: 57.0$ & 1.0 & $11.2 \times 9.7 @-46$ & 29.5 & 4.4 \\
\hline \multirow[t]{2}{*}{ Sgr D } & $17: 48: 41.0$ & $-28: 01: 41.2$ & 0.1 & 1.7 × $1.0 @ 69$ & -16.4 & 0.6 \\
\hline & $17: 48: 41.9$ & $-28: 01: 48.2$ & 0.4 & $7.5 \times 4.3 @ 60$ & -14.9 & 1.0 \\
\hline
\end{tabular}

Table 6. $\mathrm{N}_{2} \mathrm{H}^{+}$source properties.

\begin{tabular}{lllrrl}
\hline \hline Target & Significant maxima & Clumps & $\begin{array}{l}\langle v\rangle_{\mathrm{SD}} \\
\mathrm{km} \mathrm{s}^{-1}\end{array}$ & $\begin{array}{r}\sigma_{\mathrm{SD}}(v) \\
\mathrm{km} \mathrm{s}^{-1}\end{array}$ & $\begin{array}{l}\operatorname{Min}\left(\sigma_{\operatorname{Int}}[v]\right) \\
\mathrm{km} \mathrm{s}^{-1}\end{array}$ \\
\hline Sgr C & 7 & 1 & -52.5 & 6.5 & 1.1 \\
$20 \mathrm{~km} \mathrm{~s}^{-1}$ & 3 & 1 & 12.5 & 10.2 & 2.2 \\
$50 \mathrm{~km} \mathrm{~s}^{-1}$ & 9 & 1 & 48.6 & 13.9 & 1.1 \\
G0.253+0.016 & 10 & 5 & 24.9 & 16.4 & 0.6 \\
Sgr B1 off & 4 & 2 & 31.1 & 13.1 & 0.9 \\
Sgr D & 4 & 2 & -16.1 & 2.5 & 1.5 \\
\hline
\end{tabular}

Notes. Subscripts "SD" refer to single-dish observations.

only report the number of significant local maxima in Table 6. We find a significant variation between clouds. These numbers essentially characterize the complexity of emission on relatively small $p-p-v$ scales.

We then use these data to obtain information on the narrowest line widths prevailing in a given cloud. We manually search the spectra towards the $p-p-v$ maxima to find for every cloud the most narrow lines that are well-separated from other velocity components. In a further step we characterize these spectra via multi-component Gaussian fits of the sort illustrated in Fig. 4. The velocity dispersions found for the narrowest components, $\min \left(\sigma_{\text {Int }}[v]\right)$, are listed in Table 6 . Relatively narrow lines with velocity dispersions in the range 0.6 to $2.2 \mathrm{~km} \mathrm{~s}^{-1}$ are found in all CMZ clouds studied here. Kauffmann et al. (2013b) and Rathborne et al. (2015) initially reported such narrow lines for G0.253+0.016. Now we can consider the prevalence of small line widths on small spatial scales a general feature of all CMZ clouds.

The resulting velocity dispersions can be compared to the value expected for one-dimensional thermal motions of a particle of mass $m$ in gas at a temperature $T_{\text {gas }}$,

$\sigma_{\text {th }, m}(v)=288 \mathrm{~ms}^{-1}\left(\frac{m}{m_{\mathrm{H}}}\right)^{-1 / 2}\left(\frac{T_{\mathrm{gas}}}{10 \mathrm{~K}}\right)^{1 / 2}$

(where $m_{\mathrm{H}}$ is the hydrogen mass). For $\mathrm{N}_{2} \mathrm{H}^{+}$molecules with $m=29 m_{\mathrm{H}}$ at gas temperatures of 50 to $100 \mathrm{~K}$, as representative for the CMZ (see Sect. 1), one obtains thermal velocity dispersions of 0.11 to $0.17 \mathrm{~km} \mathrm{~s}^{-1}$. This means that non-thermal "turbulent" random gas motions dominate the observed velocity dispersions. However, more relevant for the gas dynamics is the comparison to the gas velocity dispersion for the mean free particle with a mass $\langle m\rangle=2.33 m_{\mathrm{H}}$ (see Appendix A of Kauffmann et al. 2008). This gives 0.4 to $0.6 \mathrm{~km} \mathrm{~s}^{-1}$ for the 50 to $100 \mathrm{~K}$ temperature range. This means that the non-thermal gas motions become similar to the thermal motions of the mean free particle. In other words, the one-dimensional sonic Mach number

$\mathcal{M}=\sigma_{\mathrm{obs}}(v) / \sigma_{\mathrm{th},\langle m\rangle}(v)$

determined from the observed velocity dispersion along the line of sight, $\sigma_{\mathrm{obs}}(v)$, indicates transonic to mildly supersonic gas motions instead of highly supersonic (i.e., $\mathcal{M} \gg 1$ ) ones. Specifically, observed velocity dispersions of 0.6 to $2.2 \mathrm{~km} \mathrm{~s}^{-1}$ imply $\mathcal{M}=1.5$ to 5.5 at $50 \mathrm{~K}$ gas temperature. Values lower by a factor $2^{1 / 2} \approx 1.4$ apply for temperatures of $100 \mathrm{~K}$. For comparison, for $50 \mathrm{~K}$ gas temperature we obtain much larger sonic Mach numbers in the range $\mathcal{M}=15$ to 40 from single-dish velocity dispersions $\sigma_{\mathrm{SD}}(v)$ as listed in Table 6.

\subsection{Steep linewidth-size relation}

The combination of interferometer maps and single-dish observations with a multi-scale characterization of cloud kinematics permits us to construct the linewidth-size relation for the CMZ. Here we construct the first such relation that extends from spatial scales $\sim 0.1$ pc to radii $>1$ pc (see Rathborne et al. 2015 for a multi-tracer exploration that characterizes scales $\geq 0.5 \mathrm{pc}$ in a single cloud).

Figure 7 collects the information on gas kinematics obtained above. The velocity dispersions from the single-dish spectra are combined with the cloud size measurements from Herschel given in Table 1. The "clumps" refer to the larger cloud 


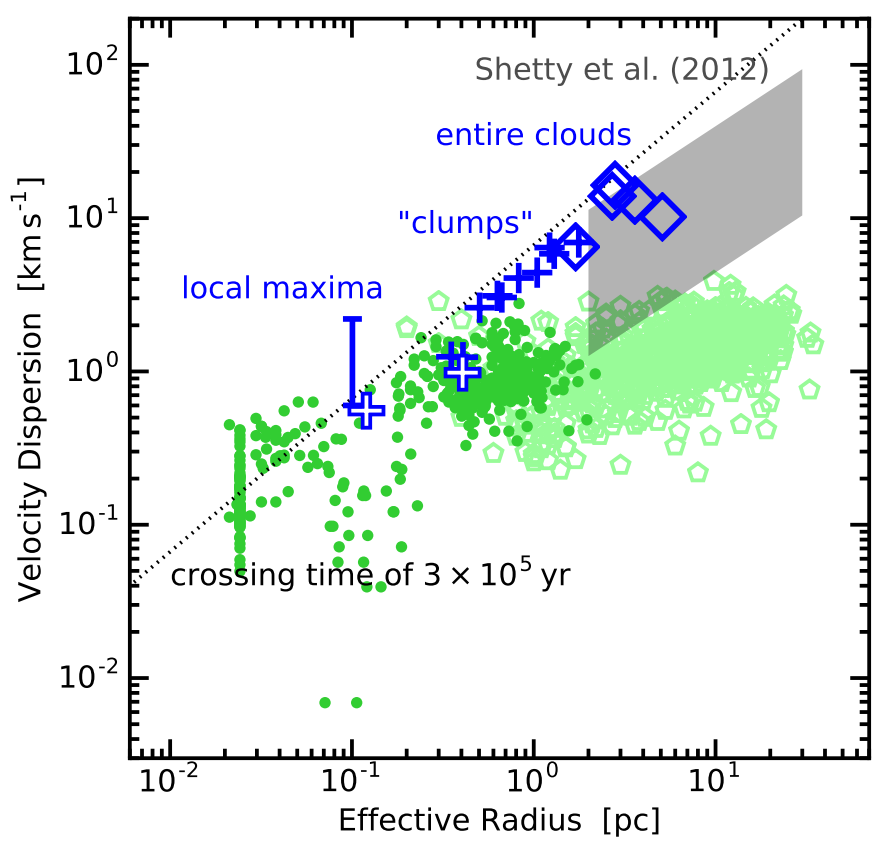

Fig. 7. Summary of the $\mathrm{N}_{2} \mathrm{H}^{+}$-based linewidth-size data explored in this paper. Blue symbols give characteristics of structures of varying spatial size explored here; see Sect. 3.4 for terminology and details. The blue crosses with white filling indicate data for the Sgr D region that probably resides outside the CMZ as studied here. The gray shaded region summarizes the linewidth-size measurements reported by Shetty et al. (2012). Dark green bullets indicate the properties of reference Milky Way clouds from the Kauffmann et al. (2013a) compilation that is based on emission lines of $\mathrm{N}_{2} \mathrm{H}^{+}$and $\mathrm{NH}_{3}$ that trace dense gas. Light green diamonds give CO-based data for the lower density gas in Milky Way clouds reported in the same collection. The dotted line is defined by a crossing time of $3 \times 10^{5} \mathrm{yr}$.

structures identified in the dendrogram analysis. The vertical bar indicates the range of minimum velocities found in the clouds by fitting spectra towards individual local $p-p-v$ maxima. A radius of 2 ".5 (i.e., $0.1 \mathrm{pc}$ ) is adopted for these structures, roughly corresponding to the beam radius of the observations. Figure 7 also includes information on CMZ gas kinematics inferred by Shetty et al. (2012; i.e., a factor 3 around the $\mathrm{N}_{2} \mathrm{H}^{+}$results in their Table 2) and the non-thermal kinematics of Milky Way molecular clouds inferred from $\mathrm{CO}$ and dense gas tracers compiled in Kauffmann et al. (2013a).

We note that the measurements of size and velocity dispersion used in Fig. 7 suffer from negligible uncertainties due to noise and imaging artifacts. We have tested this by (i) repeating the measurements in data sets to which we add artificial noise at the observed level. The measurements change at the level of a few percent between different initializations of the random noise. We have also (ii) examined results for dendrogram-based extractions where the min_delta parameter was set to $1 \mathrm{~K}$, that is, the maximum level of the corrugations seen in our images. These experiments show that the structure segmentation is not influenced by the imaging artifacts. Figure 7 does therefore not include uncertainty bars.

We recover the well-known trend that CMZ molecular clouds have very high velocity dispersions when explored on large spatial scales. For example, CMZ gas velocity dispersions measured on about $5 \mathrm{pc}$ scale exceed the values for clouds elsewhere in the Milky Way by about an order of magnitude. However, we also find that low velocity dispersions on small spatial scales are prevalent in all clouds. It appears that the observed CMZ gas motions are at the upper end of what is usually observed for spatial scales $\sim 0.1 \mathrm{pc}$ elsewhere in the Milky Way, but the dense gas kinematics in the $\mathrm{CMZ}$ are not entirely different from what is found in regular Milky Way clouds.

A very surprising trend is the steep linewidth-size relation seen in the data. We fit the observed relation by a law

$\sigma(v)=\sigma_{0}\left(r_{\mathrm{eff}} / \mathrm{pc}\right)^{h_{\sigma(v)}}$

in order to obtain a quantitative description. Specifically, we minimize the mean squared residuals between the base 10 logarithm of the observed and predicted velocity dispersions. Our analysis does not yield good estimates for the systematic uncertainties of our observations of velocity dispersion. Uncertainties in $h_{\sigma(v)}$ are therefore estimated as follows. We ignore linewidthsize data on the clumps for this analysis, since these data anyway lie between those for entire clouds and local maxima and thus add little additional information. We then vary the observed velocity dispersion on the smallest spatial scales in the range 0.6 to $2.2 \mathrm{~km} \mathrm{~s}^{-1}$, that is, between the extremes of the observed values. We assume that these two fits bracket the true range of linewidth-size relations. This gives

$\sigma(v)=(5.5 \pm 1.0) \mathrm{km} \mathrm{s}^{-1}\left(r_{\mathrm{eff}} / \mathrm{pc}\right)^{0.66 \pm 0.18}$

as the best fit. Interestingly, Shetty et al. (2012) report a similar value of 0.67 for this slope, based on the $\chi^{2}$-minimization of measurements from sigle-dish data, but their confidence range spans from 0.41 to 1.13 and is much larger than ours (arguably due to a more consequent treatment of uncertainties).

We find that the observed linewidth-size slope is unusually steep, compared to other studies of Milky Way clouds. It is not straightforward to establish this trend: one issue is that different data sets are processed in different ways. A reasonable reference is given by the collection of cloud kinematics in Kauffmann et al. (2013a) which focusses on tracers of dense gas similar to what is done here. That study yields $\sigma(v) \propto r_{\text {eff }}^{0.32}$, which is much flatter than the relation found here. Kauffmann et al. (2013a) does not list an uncertainty for the slope since that was not needed in that work. Still, it seems to be plausible that the linewidth-size relation for the CMZ is by a significant margin steeper than the relation for the dense gas in other Milky Way clouds.

Another reference point for linewidth-size relations is the classical $\sigma(v) \propto r_{\text {eff }}^{1 / 2}$ relation for entire clouds traced in $\mathrm{CO}$, as inspired by the Larson (1981) study. The modern formulation, including the exponent, is derived in a combination of observational and theoretical arguments (e.g., Heyer \& Dame 2015). This leaves the uncertainties applying to this relationship somewhat unclear. However, purely observational determinations ranging from 0.4 to 0.6 (e.g., Sanders et al. 1985; Solomon et al. 1987; Heyer \& Brunt 2004) bracket this trend and suggest an uncertainty of \pm 0.1 for the slope. These findings suggest that the CMZ linewidth- size relation does not significantly deviate from what is found for regular Milky Way clouds.

Still, it is apparent from Fig. 7 that the velocity dispersions of CMZ clouds significantly exceed those of regular Milky Way clouds for radii $\gtrsim 1 \mathrm{pc}$, while no clear difference is apparent at radii $\sim 0.1 \mathrm{pc}$. We note that this analysis combines data generated from tracers that are potentially very different. While the small scales of regular Milky Way clouds are characterized using tracers of dense gas similar to those we use for the CMZ (i.e., $\mathrm{N}_{2} \mathrm{H}^{+}$ and $\mathrm{NH}_{3}$; dark blue bullets), the largest scales are probed using $\mathrm{CO}$ (light blue diamonds), that is, a molecule that traces gas at 
densities much below those prevailing in the CMZ. It is, however, generally observed that $\mathrm{CO}$-based velocity dispersions significantly exceed those of $\mathrm{N}_{2} \mathrm{H}^{+}$and $\mathrm{NH}_{3}$ (e.g., Kirk et al. 2010). We can thus confidently claim that $\mathrm{CMZ}$ clouds explored at radii $\gtrsim 1$ pc clearly have higher velocity dispersions than regular Milky Way clouds.

In summary the data in Fig. 7 indicate that the linewidth-size relation of CMZ clouds is unusually steep by a significant factor. We stress that our claims about an unusually steep linewidthsize relation in $\mathrm{CMZ}$ clouds chiefly rest on the manual inspection of the data points shown in Fig. 7. We feel that this direct comparison suffers from fewer uncertainties than the quantitative discussion of linewidth-size fits to data sets. This unusually steep relation might have important implications for the nature of turbulence in CMZ clouds (Sect. 5.1).

That said, we caution that Shetty et al. (2012) find that the linewidth-size slope does not change between the CMZ and the solar neighborhood. This is in particular remarkable because their linewidth-size slope of 0.67 for $\chi^{2}$-minimized $\mathrm{N}_{2} \mathrm{H}^{+}$ data is similar to our result. The Shetty et al. results are in agreement with previous investigations by Oka et al. (1998) Miyazaki \& Tsuboi (2000) who also find that the kinematics of CMZ molecular clouds deviate from those of regular Milky Way clouds only by having a larger intercept, $\sigma_{0}$. However, our study differs from the Shetty et al. investigation in that we can compare clouds inside and outside the CMZ at the same spatial scale. We thus deem our results to be more robust than the conclusions provided by Shetty et al. Oka et al. do not cover a large range of spatial scales, and so they can only constrain $\sigma_{0}$. Miyazaki \& Tsuboi, by contrast, conduct an analysis very similar to ours, that is, they compare $\mathrm{CMZ}$ regions with regular Milky Way clouds over a range of spatial scales in their Fig. 2b. It is thus interesting that their analysis only indicates differences in $\sigma_{0}$, while the slope appears to be identical everywhere in the Milky Way. A major difference between their and our work, though, is that we can access spatial scales $\lesssim 0.1$ pc. The difference between our and their work could thus be explained if the linewidth-size relation steepens on small spatial scales.

The data in Fig. 7 also allow to obtain the flow crossing time, $t_{\text {cross }}=\ell / \sigma(v)$. Undriven turbulence is supposed to decay as $\mathrm{e}^{-t / t_{\text {cross }}}$ (Mac Low \& Klessen 2004), and so $t_{\text {cross }}$ also indicates the time scale on which turbulence might decay. The actual decay time might exceed $t_{\text {cross }}$, however, if the medium has clumped up into cloud fragments with small volume filling factor that interact little with another. We adopt $\ell=2 r_{\text {eff }}$ to indicate where $t_{\text {cross }}=0.3 \mathrm{Myr}$ in Fig. 7 . The velocity dispersions obtained for the local $\mathrm{N}_{2} \mathrm{H}^{+}$intensity maxima are above this relation, and they give values as low as $t_{\text {cross }}=9 \times 10^{4} \mathrm{yr}$. The kinematics for the larger cloud structures are below the reference relation, implying $t_{\text {cross }}>0.3 \mathrm{Myr}$. These measurements set a reference time scale that can, for example, be used for our later discussion of star formation processes.

\section{Suppression of CMZ star formation}

As explained in Sect. 1, it has been known for decades that star formation in the dense gas of the CMZ is suppressed by an order of magnitude, compared to clouds elsewhere in the Milky Way. Longmore et al. (2013a) quantify this trend comprehensively and provide a general framework in which the suppression of SF can be discussed. Previous studies establish that

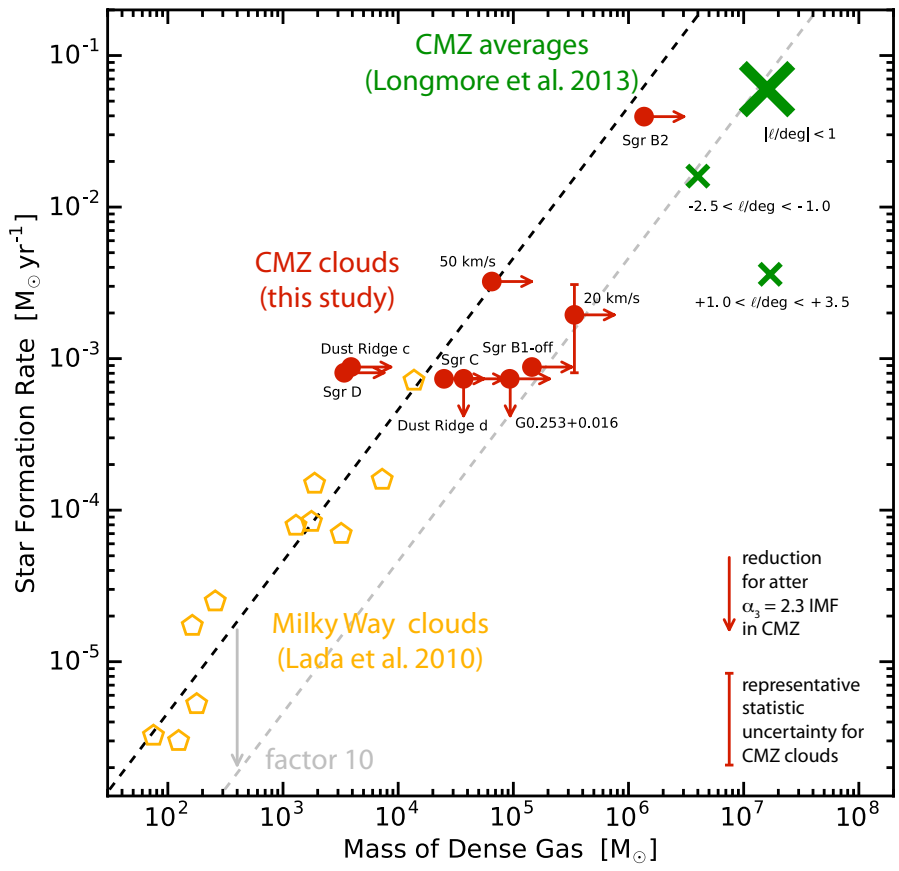

Fig. 8. Observed star-formation rates vs. the mass of dense gas residing at visual extinctions $A_{V}>7 \mathrm{mag}$. Yellow symbols give the properties of molecular clouds within about $500 \mathrm{pc}$ from sun compiled by Lada et al. (2010). Green crosses plot data for several degree-sized regions in the CMZ from Longmore et al. (2013a), as indicated by small labels. The large green cross applies to the $|\ell| \leq 1^{\circ}$ region largely explored by this paper. The red bullets give masses and star-formation rates for individual CMZ clouds determined in this paper building on data for methanol masers and $\mathrm{H}$ II regions. The red error bar in the lower right corner indicates the statistical uncertainty in SF rate that holds for the CMZ clouds containing few H II regions or none. This excludes Sgr B2, for which much lower statistical uncertainties apply. The nearby red arrow indicates the reduction in SF rate for the same clouds with $\mathrm{H}$ II regions if a flatter IMF is adopted. The black dashed line indicates a fit to the solar neighborhood data taken from Lada et al. (2010). The gray dashed line gives a relation with a star-formation rate lower by a factor 10 .

the CMZ as a whole produces fewer stars than one would expect based on the integral gas properties. In Kauffmann et al. (2013b) we demonstrate this suppression quantitatively for a single individual cloud. Figure 8 extends this analysis to include all major molecular clouds in the CMZ. The discussion presented here thus constitutes a significant update to the analysis in Kauffmann et al. (2013b). First, we now build on own welldefined mass measurements for CMZ molecular clouds, while earlier we had to compile estimates from the literature. Second, we now consider the stellar initial mass function in more detail.

This discussion requires a joint analysis of information on both the dense gas in clouds and the star formation activity. The more technical details are presented in Appendices B and C. Here we aim to summarize the discussion presented there.

\subsection{Observations of star formation in $C M Z$ clouds}

Few systematic studies of star formation in CMZ clouds exist. For example, to our best knowledge there is no reasonably complete and reliable compilation that would catalogue $\mathrm{H}$ II regions as reliable signposts of high-mass star formation. We therefore collect this information from specific studies of selected target clouds. Such observations are typically conducted with the VLA. We build on work by Liszt (1992) and 
Table 7. Summary of the star formation activity in the clouds.

\begin{tabular}{lllll}
\hline \hline Cloud & Methanol masers & Radio continuum \& spectral type & Main reference & $\dot{M}_{\mathrm{SF}} /\left(10^{-3} M_{\odot} \mathrm{yr}^{-1}\right)$ \\
\hline Sgr C & 2 & one source & Forster \& Caswell (2000) & 0.8 \\
$20 \mathrm{~km} \mathrm{~s}^{-1}$ & 0 & $1 \times \mathrm{O} 9$ & Lu et al. (2015) & $0.8-3.1$ \\
$50 \mathrm{~km} \mathrm{~s}^{-1}$ & 0 & $1 \times \mathrm{O} 7,2 \times \mathrm{O} 8.5,1 \times \mathrm{O} 9$ & Mills et al. (2011) & 3.2 \\
G0.253+0.016 & 0 & none & Immer et al. (2012a) & $<0.8$ \\
Dust Ridge C & 1 & none & Immer et al. (2012a) & $\sim 0.9$ \\
Dust Ridge D & 0 & none & Immer et al. (2012a) & $<0.8$ \\
Sgr B1-off & 1 & none & Mehringer et al. (1993) & $\sim 0.9$ \\
Sgr B2 & 11 & $20 \times \mathrm{B} 0,20 \times \mathrm{O} 9.5$ to O7, & Gaume et al. (1995) & 36 \\
& & $9 \times \mathrm{O} 6.5$ to O5 & & 0.8 \\
Sgr D & 0 & $1 \times \mathrm{O} 7$ & Liszt (1992) & \\
\hline
\end{tabular}

Liszt \& Spiker (1995) at 1.6 GHz $(\sim 19 \mathrm{~cm})$, and a series of papers by Mehringer et al. $(1993,1998)$ at wavelength between 3 and $20 \mathrm{~cm}$. Smaller fields were investigated by Ho et al. (1985) at 2 and $6 \mathrm{~cm}$, Gaume et al. (1995) at $1.3 \mathrm{~cm}$, Mills et al. (2011) at $8.4 \mathrm{GHz}(3.6 \mathrm{~cm})$, Immer et al. $(2012 \mathrm{a})$ at $8.4 \mathrm{GHz}(3.6 \mathrm{~cm})$, and Rodríguez \& Zapata (2013) at 1.3 and $5.6 \mathrm{~cm}$. In addition, for the Sgr C region we rely on the focussed study by Kendrew et al. (2013), who in part base their work on radio continuum data from Forster \& Caswell (2000).

The radio continuum observations reveal a wealth of structure including filaments that are not in any obvious way related to star formation, supernova remnants (SNRs), as well as H II regions of all kinds of sizes (i.e., from $>1 \mathrm{pc}$ to $\ll 1 \mathrm{pc}$ ). It is therefore prudent to inspect and discuss regions individually. This is done in Appendix B.

The situation is better for class II methanol $\left(\mathrm{CH}_{3} \mathrm{OH}\right)$ masers. Here a systematic study exists in the form of the methanol Multibeam Survey that employs the Parkes and ATCA observatories (Caswell et al. 2010). The data are less complex than the radio continuum images and are thus easier to interpret. Class II methanol masers are believed to trace high-mass star formation. However, since maser emission usually involves a fortuitous viewing geometry, it is not clear what fraction of embedded young high-mass stars is associated with these masers. In other words, the presence of a class II methanol maser is a sufficient marker of an embedded high mass protostar, but not a necessary one. Thus the use of class II methanol masers as tracers of star formation might be limited.

Water masers, for example near $22 \mathrm{GHz}$ frequency, provide yet another probe to detect embedded star formation. However, only the search by Lu et al. (2015) achieves a sensitivity of order $10 \mathrm{mJy}$ that is needed to reveal a significant population of the embedded masers. Future deep searches with the VLA have the potential to change this picture.

We summarize our results in Table 7 . We list only the most relevant reference for each cloud and tracer. The methanol masers are always taken from Caswell et al. (2010). We consider clouds and methanol masers to be associated only if the maser resides inside the $9 \times 10^{22} \mathrm{~cm}^{-2}$ column density contour used to trace cloud boundaries. Detailed descriptions of the data for every cloud are provided in Appendix B.

Figure 2 demonstrates that most masers are clearly associated with compact and dense structures seen in dust emission. This is expected since the masers should trace the youngest stars that are still deeply embedded in their birth environments. The H II regions, by contrast, are typically offset from the denser cloud material. This is consistent with the idea that the H II regions trace a more evolved stellar population.

\subsection{Estimates of the star-formation rate}

The observations of star formation reviewed above only constrain the presence of high-mass stars in the target clouds. But the presence, or absence, of high-mass stars hints at the total stellar population embedded in the clouds. This holds since the relative numbers of stars of low and high mass are related via the initial mass function (IMF). Appendix $\mathrm{C}$ explains how these observations can be combined with lifetime estimates of young stars to calculate star-formation rates. Here we provide a summary of that discussion. Our discussion assumes a star-formation rate of $<0.06 M_{\odot} \mathrm{yr}^{-1}$ for the region $|\ell| \leq 1^{\circ}$ as derived by Longmore et al. (2013a) using free-free foregrounds extracted from WMAP data.

In this study we adopt the Kroupa (2002) IMF with $\alpha_{3}=2.7$. Consider a stellar cluster that is described by this IMF. Fitting of the Kroupa results with a power law shows that the number of cluster members down to a mass of $0.01 M_{\odot}, N_{\mathrm{cl}}$, depends on the mass of the most massive star in the cluster, $M_{\max }$, as

$N_{\mathrm{cl}} \approx 20.5 \cdot\left(M_{\max } / M_{\odot}\right)^{1.7}$

We adopt the mean stellar mass $\left\langle m_{*}\right\rangle=0.29 M_{\odot}$ that holds for the Kroupa (2002) IMF with $\alpha_{3}=2.7$. Here we use these relationships to estimate the total mass of the young stars embedded in a cloud as $\left\langle m_{*}\right\rangle \cdot N_{\mathrm{cl}}$. We thus need to determine $N_{\mathrm{cl}}$.

$\mathrm{H}$ II regions contain central stars of spectral types 09.5 or earlier. These have stellar masses $\geq 19 M_{\odot}$ following Table 4 of Weidner \& Vink (2010). For a given cloud we thus use Eq. (4) to predict the number of stars required to form one $\mathrm{H}$ II region. We then multiply the result with the number of embedded H II regions to estimate the population of young stars associated with the cloud. Similarly, we assume $M_{\max } \sim 20 M_{\odot}$ in case class II methanol masers are detected in clouds devoid of H II regions, and we obtain the total population by multiplying the result from Eq. (4) with the number of masers. In both cases the estimated number of embedded stars is multiplied with $\left\langle m_{*}\right\rangle$ to find the mass of the embedded stellar population.

This total mass of the stellar population must be divided by some time scale in order to obtain a star-formation rate. In Appendix $\mathrm{C}$ we discuss that the association of $\mathrm{H}$ II regions and clouds must last for $\tau_{\mathrm{H} \mathrm{II}}>1.1 \mathrm{Myr}$ to explain the number of $\mathrm{H}$ II regions associated with clouds. This allows us to calculate the star-formation rate as $\dot{M}_{\mathrm{SF}}=\left\langle m_{*}\right\rangle \cdot N_{\mathrm{cl}} /(1.1 \mathrm{Myr})$. This is probably an upper limit to the true rate, given that we only have a lower limit for $\tau_{\mathrm{H} \text { II }}$.

We stress that the factors entering the calculation of the starformation rate depend on the IMF. Specifically, the product $\left\langle m_{*}\right\rangle$. $N_{\mathrm{cl}}$ will be lower by a factor 3.2 for given $M_{\max }$ if if we chose 
$\alpha_{3}=2.3$, a value that resembles the Salpeter (1955) result. The resulting star-formation rate would be lower by the same factor.

We essentially derive the star-formation rate via multiplication of some reference value with the number of $\mathrm{H}$ II regions or masers. The uncertainty in these sample sizes is governed by Poisson statistics, as detailed in Appendix C. Given the small sample sizes, this typically yields uncertainties by a factor two from the inferred SF rates. This representative uncertainty is indicated in Fig. 8.

An independent estimate of the star-formation rate can be obtained for the $20 \mathrm{~km} \mathrm{~s}^{-1}$ cloud. This region contains between 13 and 18 water masers (Lu et al. 2015). In Appendix $C$ we argue that these masers have lifetimes of order $0.16 \mathrm{Myr}$ and are powered by stars with mass $\gtrsim 2.75 M_{\odot}$. This yields $\dot{M}_{\mathrm{SF}}=$ $3 \times 10^{-3} M_{\odot} \mathrm{yr}^{-1}$.

We note that upper limits on the star-formation rate are derived for clouds that do not contain any evidence of embedded high-mass stars. This follows from the fact that the non-detection of high-mass stars sets an upper limit on $M_{\max }$ and thereby $N_{\mathrm{cl}}$.

We also note that some very young high-mass stars might not manifest as H II regions since they are "bloated" and have low surface temperatures (Hosokawa \& Omukai 2009). This effect will not significantly affect our estimates of star-formation rates, however, since we estimate the key parameter $\tau_{\mathrm{H} \text { II }}$ directly from the data.

\subsection{SF suppression in $C M Z$ clouds}

Figure 8 collects the star-formation rates calculated above. These rates are combined with Herschel-based cloud masses above a column density threshold of $9 \times 10^{22} \mathrm{~cm}^{-2}$. It is instructive to compare these rates to values determined elsewhere in the Milky Way. Reference data for this comparison come from Lada et al. (2010). They show that molecular clouds within $\sim 500$ pc from sun obey a reference relation for star-formation rate per unit dense gas of

$\dot{M}_{\mathrm{SF}, \mathrm{ref}}=(4.6 \pm 2.6) \times 10^{-8} M_{\odot} \mathrm{yr}^{-1} \cdot\left(M_{\text {dense }} / M_{\odot}\right)$.

The mass of dense gas in this analysis, $M_{\text {dense }}$, is taken to be the cloud mass enclosed by an $\mathrm{H}_{2}$ column density threshold of $6.7 \times 10^{21} \mathrm{~cm}^{-2}$ that corresponds to a visual extinction $A_{V}=$ 7 mag. The Lada et al. (2010) data and the relation describing these observations are shown in Fig. 8. Longmore et al. (2013a) use WMAP radio continuum maps obtain average star-formation rates for several regions in the $\mathrm{CMZ}$, and they use observations of dust emission to obtain measures of dense gas. These averages for three large regions in the CMZ are also shown in Fig. 8.

We note that our Herschel-based mass estimates adopt a threshold of $9 \times 10^{22} \mathrm{~cm}^{-2}$ to trace outer cloud boundaries. This is much higher than the threshold of $6.7 \times 10^{21} \mathrm{~cm}^{-2}$ which Lada et al. (2010) use to deliminate their reservoirs of dense gas. The CMZ mass reservoirs of dense gas obtained here should therefore be considered to be lower limits to the true reservoir of dense gas. That said, we believe that very little gas below the threshold of $9 \times 10^{22} \mathrm{~cm}^{-2}$ is directly associated with the clouds considered here. It is plausible that the estimates of the dense gas mass reservoirs are correct within a factor $\ll 10$.

Figure 8 shows that a number of individual CMZ clouds have star-formation rates that are significantly below the reference value set by $\dot{M}_{\mathrm{SF}, \text { ref }}$. As explained above, the dense gas masses for CMZ clouds are lower limits. The lower mass limits suggest that star formation is in some CMZ clouds suppressed by factors $>10$, compared to the solar neighborhood. Other regions might form stars at a rate consistent with $\dot{M}_{\mathrm{SF}, \text { ref }}$, such as Sgr C, Sgr D, and Dust Ridge C. But this depends on how much the true mass of dense gas in these regions deviates from the lower limit measured here.

\section{Discussion}

\subsection{Linewidth-size relation, shocks, and CMZ cloud orbits}

Section 3.4 suggests that CMZ molecular clouds appear to have an unusually steep linewidth-size relation, compared to clouds elsewhere in the Milky Way. This raises the question why such a trend might prevail in CMZ clouds.

It is plausible to speculate that this unusual linewidth-size trend might be related to other peculiar features of CMZ cloud kinematics. The latter includes unusually large line widths on spatial scales $\gtrsim 1 \mathrm{pc}$ and the prevalence of prominent shocks that are for example evident in $\mathrm{SiO}$ emission. The shocks in G0.253+0.016 are, for example, discussed by Kauffmann et al. (2013b). Jones et al. (2012) show that most or all major CMZ molecular clouds are permeated by $\mathrm{SiO}$ emission. Astrochemical models suggest that such $\mathrm{SiO}$ emission requires shocks with relative velocities $\gtrsim 20 \mathrm{~km} \mathrm{~s}^{-1}$ (Guillet et al. 2009). The high line widths and shocks could be the consequence of cloud-cloud collisions (e.g., Lis et al. 2001; Menten et al. 2009). The high velocities that are apparently observed in these collisions could reasonably result from the fast orbital motions of up to $\sim 200 \mathrm{~km} \mathrm{~s}^{-1}$ in the CMZ (Molinari et al. 2011; Kruijssen et al. 2015) that can potentially lead to significant velocity differences between clouds on slightly different orbits.

Other mechanisms that could raise the velocity dispersion include: star formation feedback in the form of supernovae, stellar winds, and radiation fields; occasional activity of Sgr A* in the form of radiation and outflows; and the influence of $\sim 10^{5}$ random main sequence stars that drift through a CMZ cloud at any given moment ${ }^{11}$. These mechanisms must combine with the aforementioned cloud-cloud collisions in a way that elevates the velocity dispersions in clouds to the high observed levels.

The relatively narrow lines observed on spatial scales $\lesssim 0.1$ pc are, by contrast, consistent with expectations for any molecular cloud. Recent work by Hacar et al. $(2013,2015)$ and Henshaw et al. (2014) indicates that the dense star-forming portions of clouds are composed of "fibers" with at most thermal internal motions on spatial scales $\lesssim 0.1 \mathrm{pc}$, that is, $\sigma(v) \lesssim \sigma_{\text {th },\langle m\rangle}(v)$ so that $\mathcal{M} \lesssim 1$. We thus would expect velocity dispersions $\lesssim 0.4$ to $0.6 \mathrm{~km} \mathrm{~s}^{-1}$ on small spatial scales in our target clouds, if we evaluate $\sigma_{\mathrm{th},\langle m\rangle}(v)$ for gas temperatures between 50 and $100 \mathrm{~K}$. This is roughly in line with our observations (Sect. 3.3).

These plausible considerations give us an idea why the velocity dispersion must decrease massively between large and small spatial scales. The arguments do not tell us, though, why the velocity dispersion must decrease steeply. One can speculate that this is a consequence of the unusually high velocity dispersions affecting part of the clouds, in the sense that the dissipation of

11 The stellar number density in the CMZ at given galactocentric radius $r$ is about $n_{*}=650 \mathrm{~cm}^{-3} \cdot(r / 50 \mathrm{pc})^{-0.8}$. This number can be estimated as follows. Launhardt et al. (2002) characterize the stellar mass distribution in the CMZ. Kruijssen et al. (2015) show that this distribution can be approximated as $M=2 \times 10^{8} M_{\odot} \cdot(r / 60 \mathrm{pc})^{2.2}$. Differentiation of this relation provides the mass density as a function of $r$, while further division by the mean stellar mass $\left\langle m_{*}\right\rangle$ yields the stellar number density. A number of $\sim 10^{5}$ in a cloud results if we then adopt a typical cloud radius of $3 \mathrm{pc}$ at $r=50 \mathrm{pc}$, which is appropriate for our sample. 
energy might be particularly efficient at $\mathcal{M} \gg 1$. We are not aware of any theoretical argument supporting this case, though.

\subsection{SF is suppressed in Individual clouds}

The data summarized in Fig. 8 have an obvious but important consequence: CMZ star formation is, at least in part, suppressed because individual CMZ clouds have suppressed SF. This challenges models in which the low SF activity of the CMZ purely results from the absence of dense and massive clouds. For example, Kruijssen et al. (2014) summarize (but do not endorse) models of this kind. Here we show that numerous massive and dense clouds do exists, but that many of these clouds are for some reason not able to produce stars at the rate expected on the basis of the cloud mass and average density.

This observation suggests that mechanisms suppressing SF in given clouds are of particular importance for the CMZ. In Kauffmann et al. (2013b) we demonstrated that at least one CMZ cloud is relatively devoid of massive dense cores of $\lesssim 0.1 \mathrm{pc}$ size that could form stars in an efficient way. This trend has since been confirmed for the same cloud by, for example, Rathborne et al. (2014b, 2015) and Johnston et al. (2014). Paper II is largely dedicated to exploring this point for the first time in a large comprehensive cloud sample (i.e., the GCMS).

\subsection{Young cloud ages unlikely to explain suppressed SF}

One might speculate that a given CMZ molecular cloud evolves from an early evolutionary state with little star formation to a later one with high star formation activity. In that case the suppression of CMZ star formation in dense gas might simply result from the fact that all CMZ clouds are for some reason in an early evolutionary state before the onset of star formation. We cannot here refute this model based on a predominance of unevolved clouds. However, our data do show that the evolution of SF activity between the clouds is more gradual than one might think.

To give a specific example, here we show that the "starless" cloud G0.253+0.016 and the "mini-starburst" Sgr B2 might form about the same number of stars per unit dense gas. Sgr B2 contains at least 49 high-mass stars exciting $\mathrm{HII}$ regions, while no such object exists in G0.253+0.016. However, Sgr B2 contains about $1.4 \times 10^{6} M_{\odot}$ of dense gas, while only $9.3 \times 10^{4} M_{\odot}$ reside in G0.253+0.016 (Table 1). Therefore, while the star-formation rate indicated by the number of $\mathrm{H}$ II regions differs by a factor $\gtrsim 49$ between the clouds, the mass of dense gas in these regions also differs by a factor $\sim 15$. In other words, compared to G0.253+0.016 the Sgr B2 region does indeed produce more stars per unit dense gas - but the difference established by the observations of $\mathrm{H}$ II regions is only a factor $\gtrsim 49 / 15 \approx 3$.

This comparison suggests that the evolution of CMZ clouds with time alone is unlikely to explain the low star formation activity in the dense gas of the CMZ. Specifically, adjusting the mix between clouds resembling Sgr B2 and those similar to $\mathrm{G} 0.253+0.016$ might alter the SF activity per unit dense gas merely by a factor $\sim 3$ : this number might be significantly larger, given that we only have an upper limit to the SF rate of G0.253+0.016. However, much larger differences in the dense gas SF rates of clouds than observed now would be required to explain the suppression of CMZ star formation in dense gas by a factor $\gtrsim 10$ (Longmore et al. 2013a) via cloud evolution alone.

One could still argue that all $\mathrm{CMZ}$ clouds are in an early evolutionary stage. This requires, however, synchronization between the clouds to an unlikely extent. Consider the evolution of individual clouds. It is plausible to think that these evolve towards efficient star formation on a time scale resembling few multiples of the free-fall $\left(3 \times 10^{5} \mathrm{yr} \cdot\left[n\left(\mathrm{H}_{2}\right) / 10^{4} \mathrm{~cm}^{-3}\right]^{-1 / 2}\right.$; Kauffmann et al. 2008) and crossing time scales $\left(\sim 3 \times 10^{5} \mathrm{yr}\right.$; Sect. 3.4). All CMZ clouds must be younger than this if they feature little SF due to a low age, and so their evolution must be synchronized to on a time scale smaller than their age, $\lesssim 3 \times 10^{5} \mathrm{yr}$. It is hard to see how this should be achieved: disturbances probably travel along the CMZ cloud orbit on a time scale resembling the orbital period $\gtrsim 4$ Myr. Such a synchronization appears unlikely, unless it is induced by powerful processes affecting the entire CMZ. Activity in Sgr $\mathrm{A}^{*}$ would be a possible candidate for such far-reaching influences.

\section{Summary}

This paper introduces the Galactic Center Molecular Cloud Survey (GCMS), the first systematic study resolving all major molecular clouds in the Central Molecular Zone (CMZ) of the Milky Way at interferometer angular resolution. Here we present images of the $\mathrm{N}_{2} \mathrm{H}^{+}(3-2)$ transition and dust continuum emission near $280 \mathrm{GHz}$ frequency. These are produced by combining data from the Submillimeter Array (SMA) and the Atacama Pathfinder Experiment (APEX). We also include dust continuum images from the Herschel Space Telescope at wavelengths between 160 and $500 \mu \mathrm{m}$, as well as an extensive literature survey for star formation associated with CMZ molecular clouds.

A companion paper (Kauffmann et al. 2017; hereafter Paper II) uses the dust continuum images to study the density structure of CMZ clouds. Here we describe the sample and the observations (Sect. 2), study the cloud kinematics (Sect. 3), and research the star formation (SF) efficiency in the dense gas of individual CMZ molecular clouds (Sect. 4). This leads to the following conclusions.

- We present the first analysis of the linewidth-size relation of CMZ clouds down to spatial scales $\sim 0.1 \mathrm{pc}$. High velocity dispersions $\geq 6.5 \mathrm{~km} \mathrm{~s}^{-1}$ observed for cloud radii $\geq 1 \mathrm{pc}$ reduce to velocity dispersions of 0.6 to $2.2 \mathrm{~km} \mathrm{~s}^{-1}$ on spatial scales $\sim 0.1$ pc (Sect. 3.4, Fig. 7). This implies an unusually steep linewidth-size relation $\sigma(v) \propto r_{\text {eff }}^{0.66 \pm 0.18}$, that is, with a power law index that appears to be significantly larger than elsewhere in the Milky Way. This steep slope might be the result of the decay of fast orbit-induced large-scale motions to transonic conditions on small spatial scales (Sect. 5.1).

- Studies of the density structure and star formation activity of CMZ clouds (Sects. 4, Appendices B and C) show that the star formation activity in individual $\mathrm{CMZ}$ clouds is suppressed by an order of magnitude (Sect. 5.2) relative to star formation relations that apply to the solar neighborhood (e.g., Heiderman et al. 2010; Lada et al. 2010). This eliminates models that try to explain the suppression of CMZ star formation purely via a suppression of the formation of dense molecular clouds.

- There is no evidence to think that CMZ star formation is suppressed because a large number of $\mathrm{CMZ}$ clouds reside in an early evolutionary phase before the onset of significant SF (Sect. 5.3). For example, the star-formation rate per unit dense gas might differ by only a factor $\sim 3$ between the prototypical "starless" cloud G0.253+0.016 and the "ministarburst" in Sgr B2. Such variations between clouds, that could plausibly result from cloud evolution, are not sufficient to explain why CMZ star formation in dense gas is suppressed by a factor $\gtrsim 10$. 
Acknowledgements. We thank a thoughtful and helpful referee who provided a thorough review full of insights. We also greatly appreciate help from Diederik Kruijssen and Steve Longmore who provided detailed feedback on draft versions of the paper. These combined sets of comments helped to significantly improve the quality and readability of the paper before and during the refereeing process This research made use of astrodendro, a Python package to compute dendrograms of astronomical data (http://www . dendrograms .org/). J.K. and T.P. thank Nissim Kanekar for initiating a generous invitation to the National Centre for Radio Astrophysics (NCRA) in Pune, India, where much of this pape was written. Q.Z. acknowledges support of the SI CGPS grant on Star Formation in the Central Molecular Zone of the Milky Way.This research was carried out in part at the Jet Propulsion Laboratory, which is operated for the National Aeronautics and Space Administration (NASA) by the California Institute of Technology. AEG acknowledges support from NASA grants NNX12AI55G, NNX10AD68G and FONDECYT grant 3150570.

\section{References}

Aguirre, J. E., Ginsburg, A. G., Dunham, M. K., et al. 2011, ApJSS, 192, 4 André, P., Men'shchikov, A., Bontemps, S., et al. 2010, A\&A, 518, L102 Aniano, G., Draine, B. T., Calzetti, D., et al. 2012, ApJ, 756, 138 Ao, Y., Henkel, C., Menten, K. M., et al. 2013, A\&A, 550, A135 Bally, J. 2008, Handbook of Star Forming Regions, 4

Bally, J., Aguirre, J., Battersby, C., et al. 2010, ApJ, 721, 137

Battersby, C., Bally, J., Ginsburg, A., et al. 2011, A\&A, 535, A128

Blum, R. D., \& Damineli, A. 1999, ApJ, 512, 237

Carey, S. J., Clark, F. O., Egan, M. P., et al. 1998, ApJ, 508, 721

Caselli, P., Benson, P., Myers, P., \& Tafalla, M. 2002a, ApJ, 572, 238

Caselli, P., Walmsley, C., Zucconi, A., et al. 2002b, ApJ, 565, 331

Caswell, J. L. 1996, MNRAS, 283

Caswell, J. L., Batchelor, R. A., Forster, J. R., \& Wellington, K. J. 1983, Austr. J. Phys., 36, 401

Caswell, J. L., Fuller, G. A., Green, J. A., et al. 2010, MNRAS, 404, 1029

Chuss, D. T., Davidson, J. A., Dotson, J. L., et al. 2003, ApJ, 599, 1116

Clark, P. C., Glover, S. C. O., Ragan, S. E., Shetty, R., \& Klessen, R. S. 2013, ApJ, 768, L34

Contreras, Y., Schuller, F., Urquhart, J. S., et al. 2013, A\&A, 549, A45

Dahmen, G., Huttemeister, S., Wilson, T. L., \& Mauersberger, R. 1998, A\&A

Di Francesco, J., Johnstone, D., Kirk, H., MacKenzie, T., \& Ledwosinska, E. 2008, ApJS, 175, 277

Diehl, R., Halloin, H., Kretschmer, K., et al. 2006, Nature, 439, 45

Egan, M. P., Shipman, R. F., Price, S. D., et al. 1998, ApJ, 494, L199

Evans, N. J., Dunham, M. M., Jørgensen, J. K., et al. 2009, ApJS, 181, 321

Evans, N. J., Heiderman, A., \& Vutisalchavakul, N. 2014, ApJ, 782, 114

Forster, J. R., \& Caswell, J. L. 2000, ApJ, 530, 371

Furuya, R. S., Kitamura, Y., Wootten, H. A., Claussen, M. J., \& Kawabe, R. 2001, ApJ, 559, L143

Gaume, R. A., Claussen, M. J., de Pree, C. G., Goss, W. M., \& Mehringer, D. M. 1995, ApJ, 449, 663

Ginsburg, A., Walsh, A., Henkel, C., et al. 2015, A\&A, 584, L7

Ginsburg, A., Henkel, C., Ao, Y., et al. 2016, A\&A, 586, A50

Guillet, V., Jones, A. P., \& Pineau des Forêts, G. 2009, A\&A, 497, 145

Güsten, R., \& Downes, D. 1983, A\&A, 117, 343

Güsten, R., Walmsley, C. M., \& Pauls, T. 1981, A\&A, 103, 197

Guzmán, A. E., Sanhueza, P., Contreras, Y., et al. 2015, ApJ, 815, 130

Hacar, A., Kainulainen, J., Tafalla, M., Beuther, H., \& Alves, J. 2015, A\&A, 587, A97

Hacar, A., Tafalla, M., Kauffmann, J., \& Kovács, A. 2013, A\&A, 554, A55

Heiderman, A., Evans, N. J., Allen, L. E., Huard, T., \& Heyer, M. 2010, ApJ, 723, 1019

Henshaw, J. D., Caselli, P., Fontani, F., Jimenez-Serra, I., \& Tan, J. C. 2014 MNRAS, 440, 2860

Heyer, M., \& Dame, T. 2015, ARA\&A, 53, 583

Heyer, M. H., \& Brunt, C. M. 2004, ApJ, 615, L45

Hillenbrand, L. A. 1997, AJ, 113, 1733

Ho, P. T. P., Jackson, J. M., Barrett, A. H., \& Armstrong, J. T. 1985, ApJ, 288, 575

Hosokawa, T., \& Omukai, K. 2009, ApJ, 691, 823

Hüttemeister, S., Wilson, T. L., Bania, T. M., \& Martin-Pintado, J. 1993, A\&A, 280,255

Hüttemeister, S., Dahmen, G., Mauersberger, R., et al. 1998, A\&A, 334, 646

Immer, K., Menten, K. M., Schuller, F., \& Lis, D. C. 2012a, A\&A, 548, A120

Immer, K., Schuller, F., Omont, A., \& Menten, K. M. 2012b, A\&A, 537, A121

Johnston, K. G., Beuther, H., Linz, H., et al. 2014, A\&A, 568, A56

Jones, P. A., Burton, M. G., Cunningham, M. R., et al. 2012, MNRAS, 419, 2961

Kauffmann, J., Bertoldi, F., Bourke, T. L., Evans, N. J., \& Lee, C. W. 2008, A\&A, 487,993
Kauffmann, J., Pillai, T., Shetty, R., Myers, P. C., \& Goodman, A. A. 2010, ApJ, 712, 1137

Kauffmann, J., Pillai, T., \& Goldsmith, P. F. 2013a, ApJ, 779, 185

Kauffmann, J., Pillai, T., \& Zhang, Q. 2013b, ApJ, 765, L35

Kauffmann, J., Pillai, T., Zhang, Q., et al. 2017, A\&A, 603, A90

Kendrew, S., Ginsburg, A., Johnston, K., et al. 2013, ApJ, 775, L50

Kirk, H., Pineda, J. E., Johnstone, D., \& Goodman, A. 2010, ApJ, 723, 457

Koda, J., Sawada, T., Wright, M. C. H., et al. 2011, ApJS, 193, 19

Koepferl, C. M., Robitaille, T. P., Morales, E. F. E., \& Johnston, K. G. 2015, ApJ, 799, 53

Kroupa, P. 2002, Science, 295, 82

Kruijssen, J. M. D., \& Cooper, A. P. 2012, MNRAS, 420, 340

Kruijssen, J. M. D., Longmore, S. N., Elmegreen, B. G., et al. 2014, MNRAS, 440, 3370

Kruijssen, J. M. D., Dale, J. E., \& Longmore, S. N. 2015, MNRAS, 447, 1059

Kurono, Y., Morita, K.-I., \& Kamazaki, T. 2009, PASJ, 61, 873

Lada, C. J., Lombardi, M., \& Alves, J. F. 2010, ApJ, 724, 687

Larson, R. 1981, MNRAS, 194, 809

Launhardt, R., Zylka, R., \& Mezger, P. G. 2002, A\&A, 384, 112

Lee, E. J., Murray, N., \& Rahman, M. 2012, ApJ, 752, 146

Lis, D. C., \& Carlstrom, J. E. 1994, ApJ, 424, 189

Lis, D. C., \& Menten, K. M. 1998, ApJ, 507, 794

Lis, D. C., Menten, K. M., Serabyn, E., \& Zylka, R. 1994, ApJ, 423, L39

Lis, D. C., Serabyn, E., Zylka, R., \& Li, Y. 2001, ApJ, 550, 761

Liszt, H. S. 1992, ApJS, 82, 495

Liszt, H. S., \& Spiker, R. W. 1995, ApJS, 98, 259

Lombardi, M., Bouy, H., Alves, J., \& Lada, C. J. 2014, A\&A, 566, A45

Longmore, S. N., Rathborne, J., Bastian, N., et al. 2012, ApJ, 746, 117

Longmore, S. N., Bally, J., Testi, L., et al. 2013a, MNRAS, 429, 987

Longmore, S. N., Kruijssen, J. M. D., Bally, J., et al. 2013b, MNRAS, 433, L15

Lu, X., Zhang, Q., Kauffmann, J., et al. 2015, ApJ, 814, L18

Mac Low, M.-M., \& Klessen, R. 2004, Rev. Mod. Phys., 76, 125

Martín-Pintado, J., de Vicente, P., Fuente, A., \& Planesas, P. 1997, ApJ, 482, L45

Maschberger, T., \& Clarke, C. J. 2008, MNRAS, 391, 711

Mehringer, D. M., Palmer, P., Goss, W. M., \& Yusef-Zadeh, F. 1993, ApJ, 412, 684

Mehringer, D. M., Goss, W. M., Lis, D. C., Palmer, P., \& Menten, K. M. 1998, ApJ, 493, 274

Menten, K. M., Wilson, R. W., Leurini, S., \& Schilke, P. 2009, ApJ, 692, 47

Mills, E. A. C., \& Morris, M. R. 2013, ApJ, 772, 105

Mills, E., Morris, M. R., Lang, C. C., et al. 2011, ApJ, 735, 84

Mills, E. A. C., Butterfield, N., Ludovici, D. A., et al. 2015, ApJ, 805, 72

Misiriotis, A., Xilouris, E. M., Papamastorakis, J., Boumis, P., \& Goudis, C. D. 2006, A\&A, 459, 113

Miyazaki, A., \& Tsuboi, M. 2000, ApJ, 536, 357

Molinari, S., Swinyard, B., Bally, J., et al. 2010, A\&A, 518, L100

Molinari, S., Bally, J., Noriega-Crespo, A., et al. 2011, ApJ, 735, L33

Mottram, J. C., Hoare, M. G., Davies, B., et al. 2011, ApJ, 730, L33

Müller, H. S., Schlöder, F., Stutzki, J., \& Winnewisser, G. 2005, J. Mol. Struct., 742,215

Müller, H. S. P., Thorwirth, S., Roth, D. A., \& Winnewisser, G. 2001, A\&A, 370, L49

Muno, M. P., Baganoff, F. K., Bautz, M. W., et al. 2004, ApJ, 613, 326

Murray, N., \& Rahman, M. 2010, ApJ, 709, 424

Novak, G., Chuss, D. T., Renbarger, T., et al. 2003, ApJ, 583, L83

Oka, T., Hasegawa, T., Hayashi, M., Handa, T., \& Sakamoto, S. 1998, ApJ, 493, 730

Ossenkopf, V., \& Henning, T. 1994, A\&A, 291, 943

Ott, J., Weiß, A., Staveley-Smith, L., Henkel, C., \& Meier, D. S. 2014, ApJ, 785, 55

Palla, F., Cesaroni, R., Brand, J., et al. 1993, A\&A, 280, 599

Piazzo, L., Calzoletti, L., Faustini, F., et al. 2015, MNRAS, 447, 1471

Pierce-Price, D., Richer, J. S., Greaves, J. S., et al. 2000, ApJ, 545, L121

Pillai, T., Kauffmann, J., Tan, J. C., et al. 2015, ApJ, 799, 74

Rathborne, J. M., Longmore, S. N., Jackson, J. M., et al. 2014a, ApJ, 786, 140

Rathborne, J. M., Longmore, S. N., Jackson, J. M., et al. 2014b, ApJ, 795, L25

Rathborne, J. M., Longmore, S. N., Jackson, J. M., et al. 2015, ApJ, 802, 125

Reid, M. J., Menten, K. M., Brunthaler, A., et al. 2014, ApJ, 783, 130

Requena-Torres, M. A., Martín-Pintado, J., Rodríguez-Franco, A., et al. 2006, A\&A, 455, 971

Requena-Torres, M. A., Martin-Pintado, J., Martin, S., \& Morris, M. R. 2008, ApJ, 672, 352

Riquelme, D., Amo-Baladrón, M. A., Martín-Pintado, J., et al. 2010a, A\&A, 523, A51

Riquelme, D., Amo-Baladrón, M. A., Martín-Pintado, J., et al. 2012, A\&A, 549, A36

Riquelme, D., Bronfman, L., Mauersberger, R., May, J., \& Wilson, T. L. 2010b, 
J. Kauffmann et al.: The Galactic Center Molecular Cloud Survey. I.

A\&A, 523, A45

Rodríguez, L. F., \& Zapata, L. A. 2013, ApJ, 767, L13

Rosolowsky, E., Pineda, J., Kauffmann, J., \& Goodman, A. 2008, ApJ, 679, 1338 Salpeter, E. E. 1955, ApJ, 121, 161

Sanders, D. B., Scoville, N. Z., \& Solomon, P. M. 1985, ApJ, 289, 373

Sault, R. J., Teuben, P. J., \& Wright, M. C. H. 2006, Astronomical Data Analysis Software and Systems IV, 77, 433

Sawada, M., Tsujimoto, M., Koyama, K., et al. 2009, PASJ, 61, S209

Schmiedeke, A., Schilke, P., Möller, T., et al. 2016, A\&A, 588, 30

Schnee, S., Li, J., Goodman, A. A., \& Sargent, A. I. 2008, ApJ, 684, 1228

Schuller, F., Menten, K. M., Contreras, Y., et al. 2009, A\&A, 504, 415

Shetty, R., Beaumont, C. N., Burton, M. G., Kelly, B. C., \& Klessen, R. S. 2012 , MNRAS, 425, 720

Shirley, Y. L. 2015, PASP, 127, 299

Siringo, G., Weiss, A., Kreysa, E., et al. 2007, The Messenger, 129, 2

Siringo, G., Kreysa, E., Kovács, A., et al. 2009, A\&A, 497, 945
Solomon, P. M., Rivolo, A. R., Barrett, J., \& Yahil, A. 1987, ApJ, 319, 730 Spergel, D. N., \& Blitz, L. 1992, Nature, 357, 665

Steer, D. G., Dewdney, P. E., \& Ito, M. R. 1984, A\&A, 137, 159

Tafalla, M., Santiago-García, J., Myers, P. C., et al. 2006, A\&A, 455, 577

Taylor, G. B., Morris, M., \& Schulman, E. 1993, AJ, 106, 1978

Tsuboi, M., Handa, T., \& Ukita, N. 1999, ApJS, 120, 1

Uchida, Y., Shibata, K., \& Sofue, Y. 1985, Nature, 317, 699

Urquhart, J. S., Moore, T. J. T., Menten, K. M., et al. 2014, MNRAS, 446, 3461

Walker, D. L., Longmore, S. N., Bastian, N., et al. 2015, MNRAS, 449, 715

Weidner, C., \& Vink, J. S. 2010, A\&A, 524, A98

Yamauchi, S., Kawada, M., Koyama, K., Kunieda, H., \& Tawara, Y. 1990, ApJ, 365,532

Yusef-Zadeh, F., Hewitt, J. W., Arendt, R. G., et al. 2009, ApJ, 702, 178

Yusef-Zadeh, F., Lacy, J. H., Wardle, M., et al. 2010, ApJ, 725, 1429

Yusef-Zadeh, F., Morris, M., \& Chance, D. 1984, Nature, 310, 557

Zhang, Q., Ho, P. T. P., \& Wright, M. C. H. 2000, AJ, 119, 1345 


\section{Appendix A: Imaging of interferometer data}

Our project is made complex by the need to image extended emission with the interferometer. The mathematics of so-called zero-spacing corrections are well understood. For example, Koda et al. (2011) provide a complete imaging pipeline for this problem. This pipeline has inspired much of the processing done below, but it requires a customized version of MIRIAD. We are not aware of a well-documented and easily useable implementation of this procedure. We therefore continue to collect and document procedures at

\section{http://tinyurl.com/zero-spacing}

Other relevant discussions of this problem are provided by Kurono et al. (2009), and the IRAM technical report ${ }^{12} 2008-2$ by Rodriguez-Fernandez, Pety \& Gueth.

\section{A.1. Imaging of dust emission}

We use CASA for the imaging of dust continuum emission. We start with the spectrally averaged data produced in the MIRIAD processing laid out in Sect. 2.2. The data are then imaged and cleaned. We use a pixel size of $00^{\prime \prime} 4$ for all sources, which resolves the synthesized beams well. Multi-scale clean is employed: we use scales of $(0,5,10,15,20)$ pixels. Briggs weights of 0.5 are chosen. The cleaning threshold is set to $6 \mathrm{mJy} \mathrm{beam}^{-1}$, which is at the upper end of the image noise levels we derive below. Up to 5000 cleaning iterations are used. The data are jointly imaged and cleaned as a mosaic.

We use an iterative scheme to image and clean the data. The main goal is to reduce the presence of obvious artifacts. The presence of such structures, for example in the form of periodic ripples, is hard to quantify. We therefore optimize the scheme via visual inspection of the resulting maps. This in particular includes the number of iterations after which no significant change is obvious, and the range of angular scales for multi-scale cleaning.

In the first step of our iteration scheme we clean the entire image down to the aforementioned threshold. This image is used to obtain a model for further iterations. To do this we highlight obvious emission features in the map using a generously drawn polygon. A model is then created from the resulting image by setting the emission outside of this boundary, as well as all negative features, to zero. Four more iteration steps follow the same procedure, with the exception that cleaning is constrained to the polygon drawn around significant emission and that CASA's CLEAN uses an emission model provided via the MODELIMAGE parameter. The procedure also creates a gain image (suffix "flux" in CASA terminology) that encodes how sensitive the final image is to sources in the outer parts of the map.

In a final step we combine the image generated from the SMA data with an estimate of the extended dust emission filtered out by the interferometer data reduction. This correction uses CASA's FEATHER tool. It is based on observations from the APEX Telescope Large Area Survey of the Galaxy (ATLASGAL, Schuller et al. 2009; see Contreras et al. 2013 for data reduction details) that were done using the Large APEX Bolometer Camera (LABOCA; Siringo et al. 2007, 2009). The LABOCA observations were done at a wavelength of $870 \mu \mathrm{m}$ with a beam size of $19^{\prime \prime} 2$. We follow the formalism of Kauffmann et al. (2008) to scale the dust emission to $280 \mathrm{GHz}$, the frequency

12 Accessible at http: //WWW . iram-institute.org of the SMA observations, by assuming a dust temperature of $20 \mathrm{~K}$ and a dust opacity varying with frequency as $\nu^{\beta}$ with $\beta=1.75$ (taken from Battersby et al. 2011 based on Ossenkopf \& Henning 1994). In practice we therefore multiply the LABOCA maps by a factor 0.50 to do this conversion. The predicted dust emission map is then multiplied by the aforementioned gain image. This makes sure that both the processed single-dish file and the interferometer-only data only contain data on emission from the same area of the $\mathrm{sky}^{13}$. In a final preparatory step we make sure that the header of the processed LABOCA map states that the beam has a size equal to the original value, that is $19^{\prime \prime} .2$. FEATHER is then run on the interferometer-only map and the processed LABOCA image. We enable the "lowpassfiltersd" option in this step.

We note that the scaling of the LABOCA map to the observing frequency of the SMA introduces negligible uncertainties $\lesssim 5 \%$ in this procedure. This dust opacity law from Battersby et al. (2011) approximates tabulated values from Ossenkopf \& Henning (1994). We note that Rathborne et al. (2014b) recently commented that $\beta \sim 1.2$ to 1.5 , based on dust emission spectral energy distributions from Herschel, but they also suggest that $1 \leq \beta \leq 2$ is broadly consistent with the data. A detailed study is needed to bring clarity. However, for a dust temperature of $20 \mathrm{~K}$, experimentation shows that the scaling factor between dust intensities at $870 \mu \mathrm{m}$ and $280 \mathrm{GHz}$ deviates by $\leq 5 \%$ relative to our choice of $\beta=1.75$ in the range $1.5 \leq \beta \leq 2.0$. Our processing of Herschel data suggests dust temperatures in the range 18 to $25 \mathrm{~K}$ for our targets (Sect. 2.4), which is similar to the $20 \mathrm{~K}$ Longmore et al. (2012) find for $\mathrm{G} 0.253+0.016$. Provided $\beta=1.75$, the scaling factor deviates by $\leq 3 \%$ relative to our reference choice of $20 \mathrm{~K}$ for dust temperatures in the range of 15 to $25 \mathrm{~K}$. More significant uncertainties in imaging can arise due to calibration uncertainties of SMA and APEX data, as well as the fundamental assumptions made within FEATHER.

\section{A.2. Imaging of line emission}

We combine actual interferometer observations with synthetic data derived from single-dish observations to populate the entire $u v$-plane from its center out to the highest spatial frequencies probed by the interferometer. This requires a number of processing steps which we implement in MIRIAD; all of the tasks mentioned below are part of this package. Below we concentrate on problems with imaging and cleaning. However, we warn that much of the development work actually concerns the accurate transfer of information between MIRIAD tasks. Careful testing is crucial to guarantee that assumptions made within MIRIAD are properly taken into account. A pixel size of $1^{\prime \prime}$ is used for all maps, which is sufficient to resolve the synthesized beam size.

We first use UVAVER to resample the interferometer into specific velocity channels. A dirty map of the entire interferometer data set is produced. The single-dish data are first smoothed to the spectral resolution of the interferometer data and then resampled into the frequency channels of the interferometer data. The resulting single-dish data are scaled to the $T_{\mathrm{mb}}$-scale using the efficiencies listed on the APEX website ${ }^{14}$.

The processed single-dish data are then cleaned using a Gaussian model approximating the beam of the single-dish

\footnotetext{
${ }^{13}$ We thank Thomas Stanke from the European Southern Observatory (ESO) for making this suggestion.

${ }^{14}$ http://www. apex-telescope.org/telescope/efficiency/
} index.php 
telescope. The idea behind this step is as follows. Many structures in single-dish maps are actually rather compact features that get blurred by the beam of the single-dish telescope. We need to identify these features since the interferometer will also detect them. We also must make sure that, as far as possible, the single-dish data does not contain corrupted (that is, blurred) information on these compact structures. Careless processing can otherwise have the result that information on the same compact source is entering into the data reduction twice - that is, once uncorrupted via the interferometer observations, and once corrupted via the blurred single-dish data. We use the Steer et al. (1984) cleaning algorithm with a gain of 0.01 and a clipping level of 0.85 .

To calculate $u v$-data from the single-dish observations we first pick $10^{4}$ points in the $u v$-plane via randomized drawing. The density distribution of these points follows the Gaussian distribution resulting from a Fourier transform of the Gaussian pattern with $23^{\prime \prime} .7$ width at half power that is assumed to describe the resolution of the single-dish map. Data points in the $u v$-plane representing baselines beyond $80 \%$ of the telescope diameter are further explicitly removed. We then loop through the pointing centers of the interferometer mosaic. For every position we generate a map representing the primary beam sensitivity pattern of the interferometer observations. The single-dish map is then multiplied with this sensitivity distribution. This processed single-dish image is then used to generate synthetic visibilities at the randomized $u v$-plane sample points generated above. Experiments show that imaging of these synthetic $u v$-data recovers the original single-dish map.

We then produce a combined set of $u v$-data that contains information from both the interferometer and single-dish observations. One critical aspect is that we must down-weight the $\sim 10^{4}$ single-dish data points in order not to suppress the interferometer data. We implement this by artificially increasing the system temperature of the single-dish data. The scaling factor is obtained as described further below. The the actual data combination and weighting is performed in MIRIAD's INVERT task with the "systemp" weighting option enabled. We use up to $10^{5}$ iterations of Steer et al. (1984) cleaning with a gain of 0.01 and a clipping level of 0.85 to deconvolve the images.

We vary the weighting factor between the single-dish and interferometer data to optimize the size and shape for the dirty beam. It is hard to devise quantitative criteria to do this adjustment. We manually minimize sidelobes and select small beams via manual inspection of the dirty beam.

At this point it is critical to realize that (i) cleaning of extended emission leaves large residuals not described by clean components, and (ii) the signal in these residuals, expressed in the Jy beam ${ }^{-1}$-scale, is calibrated to the area of the dirty beam which is generally different from the area of the restoring beam. Item (i) means that we must combine the residual with the clean components to produce a map that captures all spatial scales contained in the data. Item (ii) means that we must do this carefully, that is, by scaling the data to a common calibration of the Jy beam $^{-1}$-scale. We do the latter by calibrating the residual, obtained via MIRIAD's RESTOR task run in "residual" mode, to the area of the restoring beam. First, a representative dirty beam is produced via the MOSPSF task. Second, the area contained in this beam is measured. Third, the residual is multiplied by the ratio between the area of the restore beam to the area of the dirty beam. Fourth, the clean components are convolved with the restore beam. In a final step the convolved clean components are added to the re-calibrated residual map to obtain the final data set. We find that this procedure guarantees that the resulting map recovers the original intensities to $<10 \%$ of the values seen by the single-dish telescope.

\section{Appendix B: Observations of star formation in $\mathrm{CMZ}$ clouds}

\section{B.1. $\operatorname{Sgr} C$}

Liszt \& Spiker (1995) use a $1.6 \mathrm{GHz}$ survey of the CMZ by Liszt (1992) to characterize the emission of Sgr C. The emission from this region is found to be thermal. $\mathrm{H} 70 \alpha$ recombination lines yield a velocity of $-65 \mathrm{~km} \mathrm{~s}^{-1}$. The continuum emission is rather extended, with a radius of 1' corresponding to $2.3 \mathrm{pc}$ at the distance of the CMZ; this is not a compact $\mathrm{H}$ II region. A single star of spectral type $\mathrm{O} 4$ or $\mathrm{O} 6$ could provide the required ionization.

The H II region is not directly embedded in the dust cloud as here extracted from Herschel maps. This region of 1' (i.e., $\sim 2 \mathrm{pc}$ ) radius is outside of the Herschel-derived cloud boundaries by at least $1^{\prime}$. The $\mathrm{Sgr} \mathrm{C}$ cloud appears to reside exactly at the boundary of the H II region and might have been sculpted by it. This would indicate that the molecular cloud and the H II region are indeed connected. For example, the cloud as seen today might be a leftover of the star formation process. However, there is no clear indication of a direct link, such as coincidence within the Herschel-derived boundaries. We abstain from claiming a speculative association between this large $\mathrm{H}$ II region and the Sgr C cloud.

Instead we build on a detailed study of star formation in Sgr C. Kendrew et al. (2013) demonstrate that a compact and faint H II region initially detected by Forster \& Caswell (2000) is embedded in this cloud. The radio data have not been used to determine a spectral type: here we adopt a mass of $18 M_{\odot}$ to be conservative.

\section{B.2. $20 \mathrm{~km} \mathrm{~s}^{-1}$ cloud}

The thermal radio source "G" of Ho et al. (1985) resides well inside the boundaries of the $20 \mathrm{~km} \mathrm{~s}^{-1}$ cloud. It is interpreted as a single H II region or a cluster of such sources. A single star of spectral type O9 could excite this region. Further, Lu et al. (2015) report the discovery of up to 18 water masers in this cloud.

\section{B.3. $50 \mathrm{~km} \mathrm{~s}^{-1}$ cloud}

Mills et al. (2011) provide a recent summary of this region. A chain of four previously known H II regions, labeled " $A$ " through " $D$ " from north to south, is detected. The most southern region " $\mathrm{D}$ " is the most compact and presumably youngest object. The spectral types of the exciting stars listed in Table 7 are derived assuming a single ionizing source. Yusef-Zadeh et al. (2010) use observations of $\mathrm{Ne}$ II to derive systemic velocities $\sim 40 \mathrm{~km} \mathrm{~s}^{-1}$ for the targets.

\section{B.4. $\mathrm{G} 0.253+0.016$}

The star formation status of this region is controversial. Immer et al. (2012a) find no radio continuum source inside this cloud. Their source "A" lies 1!3 outside of the Herschelderived cloud boundary. The exciting source could be a single O9 star. There is no obvious reason to think that source " $\mathrm{A}$ " from Immer et al. (2012a) was recently born in G0.253+0.016.

However, using the same data, Rodríguez \& Zapata (2013) find several additional sources in the vicinity of G0.253+0.016. 
Their source " 3 " is the object "A" discussed by Immer et al. (2012a). A radial velocity of $45 \mathrm{~km} \mathrm{~s}^{-1}$ from previous recombination line observations is quoted. Sources " 1 ", "2", and "7" have non-thermal spectral indexes indicating that these are not H II regions. Sources "4", "5", and "6" have a thermal nature. Their flux densities are each consistent with excitation by a single B0.5 star. These objects are inside the cloud boundary defined via the Herschel data, except for source " 6 " which is offset by 0 '2 . Given the sensitivities achieved by the observations, all of the targets seen by Rodríguez \& Zapata (2013) should also have been detected by Immer et al. (2012a). The most plausible explanation for this discrepancy is that Rodríguez \& Zapata detect an extended component of radio continuum emission which is not picked up in the data reduction of Immer et al. This is in particular suggested by such emission being detected in new VLA maps by Mills et al. (2015). Extended radio emission would not hint at star formation in $\mathrm{G} 0.253+0.016$ but rather would reflect the general excitation conditions in the CMZ. We therefore ignore the three sources found by Rodríguez \& Zapata (2013).

\section{B.5. Dust Ridge "C" \& “ $D$ "}

These regions are surveyed by Immer et al. (2012a). No radio sources associated with these clouds are detected.

\section{B.6. Sgr B1-off}

Mehringer et al. (1993) find a number of compact sources in the vicinity of Sgr B1-off. Their sources "A" (0'.5 offset, i.e. $1.2 \mathrm{pc})$ and "C" (0.9 offset, i.e. $2.1 \mathrm{pc})$ are those closest to the dust emission feature we extract from Herschel maps. Following Mehringer et al. (1993), single stars with spectral types B0 would be sufficient to excite these regions. However, there is no obvious reason to think that these H II regions are physically associated with Sgr B1-off.

\section{B.7. Sgr B2}

Gaume et al. (1995) detect a total of 49 compact radio continuum sources in this region. The numbers given in Table 7 assume that every source is excited by a single star.

\section{B.8. $\operatorname{Sgr} D$}

Liszt (1992) finds two structures in Sgr D. One is a supernova remnant (SNR). $\mathrm{H}_{2} \mathrm{CO}$ absorption lines at up to $+125 \mathrm{~km} \mathrm{~s}^{-1}$ are seen against this source. The other one is an $\mathrm{H}$ II region for which $\mathrm{H} 70 \alpha$ recombination lines yield a velocity of $-19 \mathrm{~km} \mathrm{~s}^{-1}$. However, the aforementioned $\mathrm{H}_{2} \mathrm{CO}$ lines are also seen against this source. The H II region contains a compact core to which Liszt (1992) refers as source "3". He derives an ionization rate consistent with a single $\mathrm{O} 7 \mathrm{star}$ for this core, if the source is located in the CMZ. Similar results were derived by Mehringer et al. (1998). This source falls directly on top of the features in our SMA maps. It is clearly associated with the Sgr D molecular cloud.

\section{Appendix C: Estimates of the star-formation rate}

The observations of star formation reviewed above only constrain the presence of high-mass stars in the target clouds. But the presence, or absence, of high-mass stars hints at the total stellar population embedded in the clouds, since the relative number of stars of low and high mass are related via the initial mass function (IMF). In this study we adopt the Kroupa (2002) IMF with $\alpha_{3}=2.7$. Consider a stellar cluster that is described by this IMF. Fitting his results with a power law shows that the number of cluster members down to a mass of $0.01 M_{\odot}, N_{\mathrm{cl}}$, depends on the mass of the most massive star in the cluster, $M_{\max }$, as

$N_{\mathrm{cl}} \approx 20.5 \cdot\left(M_{\max } / M_{\odot}\right)^{1.7}$.

We adopt the mean stellar mass of $0.29 M_{\odot}$ that holds for the Kroupa (2002) IMF with $\alpha_{3}=2.7$. Equation (C.1) permits to estimate masses for the stellar populations associated with the CMZ clouds. We now need to divide these masses by a timescale in order to obtain a star-formation rate.

\section{C.1. Lifetimes of $H I I$ regions in the $C M Z$}

Following Weidner \& Vink (2010), coeval main sequence stars can populate the spectral types of relevance here (i.e., up to $\mathrm{O} 4$ ) for 2 to $7 \mathrm{Myr}$. One could use these numbers if it were certain that the stars power H II regions throughout their lifetime. But this is not clear, in particular not in a region like the CMZ.

For example, the high velocity dispersions inside the clouds might mean that the stars produced in these regions are born with high relative velocities with respect to their natal clouds. In that case the stars powering the $\mathrm{H}$ II regions might quickly detach from their birth sites. If present, this process might occur on time scales $\gtrsim t_{\text {cross }}$, provided that velocity also controls the relative motion of stars and gas inside a cloud. The discussion in Sect. 3.4 gives crossing times $>0.3 \mathrm{Myr}$ on intermediate to large spatial scales.

Alternatively we can combine the global star formation record of the $\mathrm{CMZ}$ with the observed number of $\mathrm{H}$ II regions to constrain the timescale over which $\mathrm{H}$ II regions are associated with CMZ molecular clouds. Longmore et al. (2013a) obtain a star-formation rate of $<0.06 M_{\odot} \mathrm{yr}^{-1}$ for the region $|\ell| \leq 1^{\circ}$ using free-free foregrounds extracted from the WMAP data, when considering all radiation at $|b| \leq 1^{\circ}$. Here we consider this to be an upper limit since it is likely that radiation from Sgr $\mathrm{A}^{*}$ unrelated to the star formation process contributes to the ionizing radiation characterized in this experiment. Longmore et al. (2013a) use results from Murray \& Rahman (2010) to estimate that the high-mass stars traced by the free-free maps have a mean age $\tau_{\mathrm{HM}}=4$ Myr. Now we use Eq. (C.1) to find that 2791 stars are needed to produce one high-mass star of $18 M_{\odot}$ that can power an $\mathrm{H}$ II region. This number can be multiplied with the mean stellar mass of $0.29 M_{\odot}$ and divided by the timescale $4 \mathrm{Myr}$ to find that a star-formation rate of $2 \times 10^{-4} M_{\odot} \mathrm{yr}^{-1}$ is required to produce one star of a mass high enough to power an $\mathrm{H}$ II region. This suggests that $N_{\mathrm{HM}}<0.06 M_{\odot} \mathrm{yr}^{-1} /\left(2 \times 10^{-4} M_{\odot} \mathrm{yr}^{-1}\right) \approx 300$ such stars exist in the inner CMZ where $|\ell| \leq 1^{\circ}$. This count can be compared to the number of known H II regions in the same volume: combining data from Ho et al. (1985), Mehringer et al. (1993), Liszt \& Spiker (1995), Gaume et al. (1995), Mills et al. (2011), and Immer et al. (2012a) we find $N_{\mathrm{H} \text { II }} \geq 80$ such objects at $|\ell| \leq 1^{\circ}$. We can now make the assumption that the relative number ratio between the $\mathrm{H}$ II regions and the total count of highmass stars is equal to the ratio between the duration of the phase where $\mathrm{H}$ II regions are embedded in clouds and the mean lifetime of high-mass stars, $N_{\mathrm{H} \text { II }} / N_{\mathrm{HM}} \sim \tau_{\mathrm{H} \text { II }} / \tau_{\mathrm{HM}}$. Rearrangement and substitution gives $\tau_{\mathrm{H} \text { II }}>1.1 \mathrm{Myr}$. We consider this a lower limit because of the aforementioned contribution of Sgr A* to the ionizing radiation and the lower limit on the number of $\mathrm{H}$ II regions (from our non-systematic literature survey). 
We adopt this number of $1.1 \mathrm{Myr}$, which is also consistent with the qualitative estimate $\tau_{\mathrm{H} \text { II }} \gtrsim t_{\text {cross }}>0.3 \mathrm{Myr}$ obtained above. This value is also broadly consistent with the age of the stellar population that is immediately associated with the Orion Nebula (Bally 2008). Substitution yields $\dot{M}_{\mathrm{SF}}=0.29 M_{\odot}$. $N_{\mathrm{cl}} /(1.1 \mathrm{Myr})$.

\section{C.2. Reference relations for star formation}

A reference dense gas star-formation rate that holds for clouds near the Sun is provided by Lada et al. (2010),

$\dot{M}_{\mathrm{SF}, \text { ref }}=(4.6 \pm 2.6) \times 10^{-8} M_{\odot} \mathrm{yr}^{-1} \cdot\left(M_{\text {dense }} / M_{\odot}\right)$.

It is obtained by multiplication of the number of embedded young stellar objects, $N_{\text {YSO }}$, with a mean stellar mass of $0.5 M_{\odot}$ and division by a maximum age of young stellar objects of $2 \mathrm{Myr}$, so that $\dot{M}_{\mathrm{SF}}=0.5 M_{\odot} \cdot N_{\mathrm{YSO}} /(2 \mathrm{Myr})$. In Kauffmann et al. (2013b) we argued that this value should be increased by some factor because infrared observations only reveal a fraction of the true population of embedded young stars. Here we make the more conservative assumption that all embedded stars are counted, and we follow Evans et al. $(2009,2014)$ in adopting a mean stellar mass of $0.5 M_{\odot}$, equal to that of well-studied populations of embedded stars. Increases in $\dot{M}_{\mathrm{SF}, \text { ref }}$, as appropriate if a significant number of stars are missed, would imply that CMZ star formation is suppressed by factors larger than those obtained in the main text.

We note that some of the assumptions made in the analysis of $\mathrm{HII}$ regions are not entirely consistent with those made in the analysis of infrared samples of embedded young stars. For example, both Evans et al. (2009, 2014) and Lada et al. (2010) assume a mean stellar mass of $0.5 M_{\odot}$ where we adopt a value of $0.29 M_{\odot}$. This difference comes about because infrared surveys are not complete down to the substellar regime, while the Kroupa (2002) IMF analysis conducted above considers masses down to $0.01 M_{\odot}$. For example, the mean stellar mass of the Kroupa (2002) IMF for stellar masses $>0.1 M_{\odot}$ is $0.5 M_{\odot}$, consistent with what is adopted for the analysis of infrared surveys. However, only $8 \%$ of the stellar mass in the Kroupa (2002) IMF resides in the mass interval of 0.01 to $0.1 M_{\odot}$. One would need to lower the CMZ star-formation rates by this percentage to achieve full conceptual consistency. Here we omit this small correction due to the other systematic uncertainties involved.

\section{C.3. Data on HII regions}

The spectral types of the sources exciting H II regions indicate the masses of the illuminating stars. We adopt the mean initial mass as a function of the spectral type for dwarf stars with realistic rotation from Table 4 of Weidner \& Vink (2010) to do this calculation.

H II regions are powered by stars of spectral type O9.5 or earlier. These stars have masses $>18 M_{\odot}$ following Weidner \& Vink. Equation (C.1) can be interpreted in the sense that on average a group of 3059 stars is needed to form one star of $\geq 19 M_{\odot}$. Following $\dot{M}_{\mathrm{SF}}=0.29 M_{\odot} \cdot N_{\mathrm{cl}} /(1.1 \mathrm{Myr})$ such a population implies a star-formation rate of $8 \times 10^{-4} M_{\odot} \mathrm{yr}^{-1}$. The presence of $N_{\mathrm{H} \text { II }} \mathrm{H}$ II regions thus statistically implies a starformation rate

$\dot{M}_{\mathrm{SF}}=8 \times 10^{-4} M_{\odot} \mathrm{yr}^{-1} \cdot N_{\mathrm{HII}}$.

We use this relation to derive star-formation rates from the observational data on H II regions. This approach suffers from statistical uncertainties due to how actual star formation in a region samples the IMF. Provided $N_{\mathrm{cl}}$ is held constant, deviations between the statistically expected number of $\mathrm{HII}$ regions and the actual one are described by Poisson statistics. For these the standard deviation of a measurement with expectation value $x$ is given by $\sigma(x)=x^{1 / 2}$. This suggests to use $\sigma\left(N_{\mathrm{H} \text { II }}\right)=N_{\mathrm{H} \text { II }}^{1 / 2}$ and

$\sigma\left(\dot{M}_{\mathrm{SF}}\right)=8 \times 10^{-4} M_{\odot} \mathrm{yr}^{-1} \cdot N_{\mathrm{H} \mathrm{II}}^{1 / 2}$

to estimate the statistical uncertainties on $\dot{M}_{\mathrm{SF}}$. This implies uncertainties of order of a factor 2 for the small numbers of $\mathrm{H}$ II regions detected in most of our targets. This is the uncertainty indicated in Fig. 8. A much lower relative factor applies in Sgr B2 due to the rich sample of high-mass stars (the absolute value of the uncertainty increases, though). On top of these there are further systematic uncertainties, for example due to uncertainties in the shape of the IMF and the relationship between stellar masses and spectral types. An assessment of these possible sources of error is well beyond the scope of the current study.

Alternatively one could use the mass of the star with the earliest spectral type to obtain $N_{\mathrm{cl}}$ from Eq. (C.1) and use this number to derive the SF rate. However, the maximum mass sampled from an IMF can deviate from the expectation value by factors 2 (see black lines in Fig. 1 of Kruijssen \& Cooper 2012 for sampling from a power-law distribution function, based on a formalism by Maschberger \& Clarke 2008). Unfortunately, $N_{\mathrm{cl}}$ strongly depends on $M_{\max }$ and the mass uncertainty implies uncertainties by factors $\sim 2^{1.7}=3.25$ relative to the expectation value $^{15}$. While potentially useful, here we abstain from such estimates.

We use data on the Gould Belt molecular clouds in the solar neighborhood (i.e., $d \lesssim 500$ ) from Lada et al. (2010) to conduct a consistency check of our method. Except for Orion, none of the Gould Belt clouds contain H II regions. This implies stellar masses $\leq 18 M_{\odot}$ and therefore $\dot{M}_{\mathrm{SF}}<0.8 \times 10^{-3} M_{\odot} \mathrm{yr}^{-1}$. The latter star-formation rate is in line with the data compiled by Lada et al. (2010).

In Orion we do find high-mass stars and a major $\mathrm{H}$ II region. Hillenbrand (1997) identify five stars of mass $\geq 20 M_{\odot}$ in this region. In this case we obtain $N_{\mathrm{cl}}$ for $M_{\max }=20 M_{\odot}$ and increase that value by a factor 5 to derive the SF rate from $\dot{M}_{\mathrm{SF}}=$ $0.29 M_{\odot} \cdot N_{\mathrm{cl}} /(1.1 \mathrm{Myr})$. We find $\dot{M}_{\mathrm{SF}}=4.4 \times 10^{-3} M_{\odot} \mathrm{yr}^{-1}$. This is a bit high compared to the rate of $7 \times 10^{-4} M_{\odot} \mathrm{yr}^{-1}$ adopted by Lada et al. (2010).

\section{C.4. Data on methanol masers}

Here we assume that class II methanol masers indicate the formation of stars of $\sim 20 M_{\odot}$ mass. The $6.7 \mathrm{GHz}$ masers used here are almost exclusively associated with luminous cloud fragments that are massive enough to form high-mass stars (Urquhart et al. 2014) - but this finding gives no clear minimum stellar mass for their excitation. Here we adopt a rather high mass to be conservative (i.e., obtain upper limits to the star-formation rate).

Two of our target clouds host such masers but are devoid of $\mathrm{H}$ II regions. This might be the case because these high-mass stars are still deeply enshrouded in the cloud and are unable to ionize a detectable volume of gas. The star-formation rate listed in Table 7 is calculated assuming the aforementioned value as the maximum stellar mass. We ignore the methanol maser data in case HII regions are detected because the radio continuum data are more easily and accurately interpreted.

15 We thank D. Kruijssen for kindly pointing out this problem. 


\section{C.5. Data on water masers}

One other alternative estimate can be obtained for the $20 \mathrm{~km} \mathrm{~s}^{-1}$ region. Lu et al. (2015) present a detailed and recent study of this region. Here we can use their detections of water masers to obtain an alternative measure of the star-formation rate. Between 13 and 18 masers have line luminosities $\gtrsim 10^{-7} L_{\odot}$. We assume that every maser corresponds to one star. These line luminosities imply bolometric luminosities $\gtrsim 50 L_{\odot}$ (Palla et al. 1993), which corresponds to stellar masses $\gtrsim 2.75 M_{\odot}$ (assuming the mass-luminosity data from Mottram et al. 2011 can be interpolated towards solar values using a power-law). One such star requires a stellar group of 115 stars (Eq. (C.1)). The full maser population thus implies between 1480 and 2050 embedded stars, each with a mean stellar mass of $0.29 M_{\odot}$. Water masers are usually associated with the class 0 stage of star formation (Furuya et al. 2001) which has a duration of order $0.16 \mathrm{Myr}$ (Evans et al. 2009). Combination of these numbers gives a starformation rate $\sim 3 \times 10^{-3} M_{\odot} \mathrm{yr}^{-3}$. This is significantly in excess of the rate of $\sim 0.8 \times 10^{-3} M_{\odot} \mathrm{yr}^{-3}$ implied by the data on $\mathrm{H}$ II regions.

This estimate suffers from a number of uncertainties. First, it is not clear that every luminous young star produces water masers. This could mean that we miss a significant number of young stars. Second, a single star might produce several masers. This could imply that we overestimate the number of young embedded stars. Third, there is significant scatter in the relationship between maser and bolometric luminosities (Palla et al. 1993). Fourth, the current data on the relationship between water masers and stellar evolutionary stages is dated (Furuya et al. 2001) and not necessarily closely linked to the evolutionary stages of SF as understood and defined today (Evans et al. 2009). Fifth, much of the bolometric luminosity of lower-mass stars with water masers might come from accretion. In that case the conversion from bolometric luminosities overestimates stellar masses therefore the SF rate. Table 7 lists the SF rate determined here, but future updates appear likely. 